\title{
Fourier-based analysis and synthesis of moirés in the superposition of geometrically transformed periodic structures
}

\author{
Isaac Amidror and Roger D. Hersch \\ Laboratoire de Systèmes Périphériques, Ecole Polytechnique Fédérale de Lausanne, 1015 Lausanne, Switzerland
}

Received August 1, 1997; revised manuscript received November 25, 1997; accepted December 8, 1997

\begin{abstract}
The best method for investigating moiré phenomena in the superposition of periodic layers is based on the Fourier approach. However, superposition moiré effects are not limited to periodic layers, and they also occur between repetitive structures that are obtained by geometric transformations of periodic layers. We present in this paper the basic rules based on the Fourier approach that govern the moiré effects between such repetitive structures. We show how these rules can be used in the analysis of the obtained moirés as well as in the synthesis of moirés with any required intensity profile and geometric layout. In particular, we obtain the interesting result that the geometric layout and the periodic profile of the moire are completely independent of each other; the geometric layout of the moire is determined by the geometric layouts of the superposed layers, and the periodic profile of the moiré is determined by the periodic profiles of the superposed layers. The moiré in the superposition of two geometrically transformed periodic layers is a geometric transformation of the moiré formed between the original layers, the geometric transformation being a weighted sum of the geometric transformations of the individual layers. We illustrate our results with several examples, and in particular we show how one may obtain a fully periodic moiré even when the original layers are not necessarily periodic. (C) 1998 Optical Society of America [S0740-3232(98)00905-3]
\end{abstract}

OCIS codes: 120.4120, 080.0080, 070.2590.

\section{INTRODUCTION}

The superposition moiré is a well-known phenomenon that occurs when periodic or repetitive structures (line gratings, dot screens, etc.) are superposed. It consists of a visible pattern that is clearly observed at the superposition, although it does not appear in any of the original structures. The moiré effect between superposed structures occurs because of the geometric distribution of dark and bright areas in the superposed image: Areas in which dark elements of the original structures cross each other contain less black than areas where the original structure elements fall between each other and fill the white spaces better. The importance of this phenomenon is clearly demonstrated by its vast number of applications in many different fields. ${ }^{1-5}$

Although classical geometric or algebraic approaches can be used to explain the superposition moiré phenomenon and its geometric properties, ${ }^{6,7}$ it has been shown that the best approach for exploring phenomena related to the superposition of periodic structures is the approach based on the Fourier theory. ${ }^{8-10}$ Using the standard representation of the intensity of each layer as a reflectance (or transmittance) function $r_{i}(x, y)$ with values ranging between 0 (for black) and 1 (for white), we can express the layer superposition mathematically as the product of the $m$ individual layers:

$$
r(x, y)=r_{1}(x, y) \ldots r_{m}(x, y) .
$$

In the case of periodic layers, each of the layers $r_{i}(x, y)$ can be represented in the form of a Fourier series, and hence this case lends itself in a natural way to an exploration by means of the Fourier theory. ${ }^{10}$ However, su- perposition moiré effects are not limited to periodic layers, and they may also occur between various repetitive layers that are not necessarily periodic. ${ }^{11}$ In the present paper we will consider such structures as nonlinear geometric transformations of periodic, two-dimensional (2D) structures. We will present the basic rules that govern the geometric layout and the intensity profile of moiré effects between such repetitive structures, and we will show how these rules can be used in the analysis of the obtained moirés as well as in the synthesis of moirés that have any required intensity profile and geometric layout. In particular, we will show how our approach permits us to generalize classical results that are already known for first-order moirés into the general cases of higher-order moirés and moirés between $2 \mathrm{D}$ structures such as dot screens.

The outline of this paper is as follows: In Section 2 we introduce the basic notions about repetitive structures that are not necessarily periodic (curvilinear gratings, curved dot screens, etc.). In Section 3 we review the Fourier decomposition of such repetitive structures, and in Section 4 we present the basic notions related to the superposition of repetitive layers and to their moiré effects. The main results are presented in Section 5, first for the simpler case of curvilinear gratings and then for the more general case of curved dot screens. Section 6 concludes the paper with a short review of the main results.

\section{REPETITIVE STRUCTURES}

Under the heading of "repetitive structures" one may lump together many different types of structures, which, 
although not necessarily periodic, still show some kind of internal structural repetition that is governed by some given rules.

We will restrict ourselves in this paper to the main type of repetitive, nonperiodic structure, namely, coordinatetransformed structures. As their name indicates, each of these structures can be obtained from a certain initial periodic structure by the application of an appropriate nonlinear coordinate transformation. Curvilinear gratings, straight gratings with varying frequency, curved line grids, and curved dot screens all belong to this important category. The best way to deal with each of these structures is to consider it as a nonlinear geometric transformation of a periodic, $2 \mathrm{D}$ structure. One may think of this transformation as an operation that "bends," or nonlinearly stretches, the original periodic structure according to a given mathematical rule.

We begin with the simplest case of coordinatetransformed structures, that of curvilinear gratings. Let $r(x, y)$ (denoting the reflectance or the transmittance at location $x, y)$ be the curvilinear grating that is obtained by bending the $2 \mathrm{D}$ onefold periodic grating $p\left(x^{\prime}\right)$, i.e., by replacing $x^{\prime}$ with the function $g_{1}(x, y): r(x, y)$ $=p\left[g_{1}(x, y)\right]$ (see various examples in Fig. 1). The intensity profile of the original, uncurved periodic grating, $p\left(x^{\prime}\right)$, or sometimes its one-dimensional (1D) section along the $x^{\prime}$ axis, is called the periodic profile of the curvilinear grating $r(x, y)$. The periodic profile of a curvi- linear grating may be cosinusoidal, a square wave, a sawtooth wave, or any other periodic waveform. The function $x^{\prime}=g_{1}(x, y)$ that bends $p\left(x^{\prime}\right)$ into the curvilinear grating $r(x, y)$ is called the bending transformation. Note that $x^{\prime}, y^{\prime}$ are the coordinates of the original, periodic space, while $x, y$ are the coordinates of the target, transformed space; the bending transformation is therefore a backward mapping from the transformed coordinates to the original coordinates.

A curvilinear grating $r(x, y)=p\left[g_{1}(x, y)\right]$ is therefore characterized by two basic and independent properties:

1. Its geometric layout in the $x, y$ plane, i.e., the locus of the centers of its curvilinear corrugations in the $x, y$ plane, which is defined by the bending transformation $x^{\prime}=g_{1}(x, y)$.

2. The intensity behavior across each of the curvilinear corrugations, which is determined by the periodic profile $p\left(x^{\prime}\right)$.

Note that since in general the frequency of the curvilinear grating $r(x, y)$ varies throughout the plane, we may arbitrarily choose any frequency for the periodic profile $p\left(x^{\prime}\right)$. For the sake of convenience we will often choose this frequency to be $f=1$ [or, equivalently, we may consider $f$ to be included within $\left.g_{1}(x, y)\right]$; in such cases the term "normalized periodic profile" will be used.

Example 1: Assume that we are given a 2D cosinusoidal grating $p\left(x^{\prime}\right)=\cos \left(2 \pi f x^{\prime}\right)$ over the $x, y$ plane [Fig.

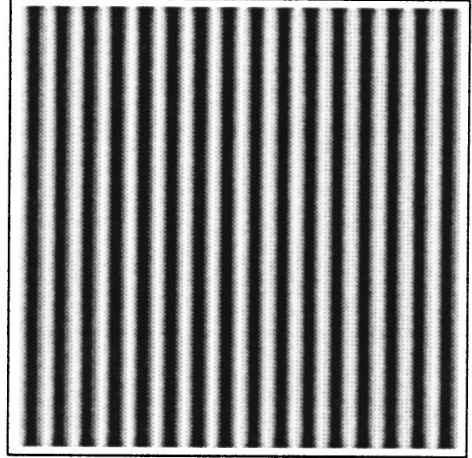

(a)

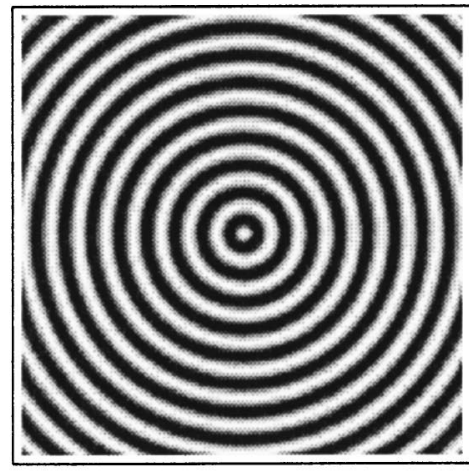

(d)

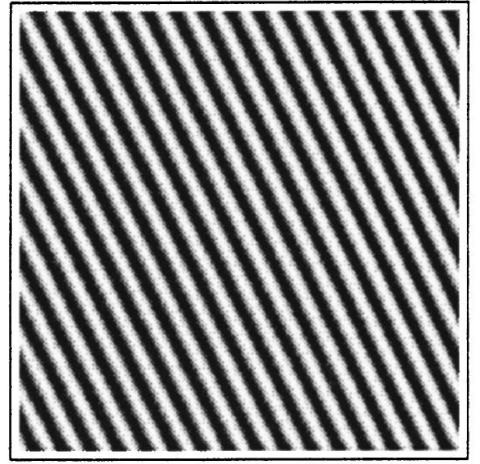

(b)

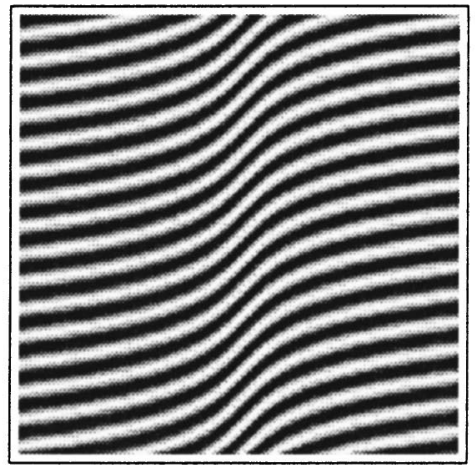

(e)

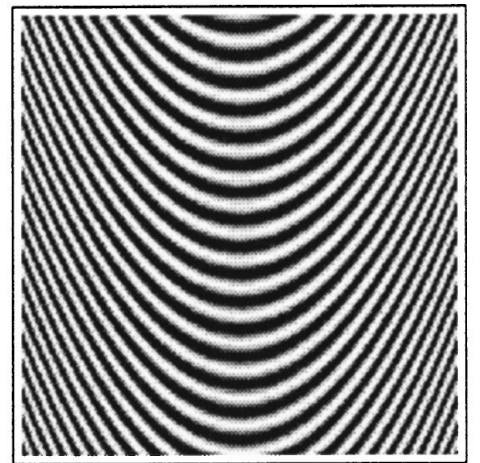

(c)

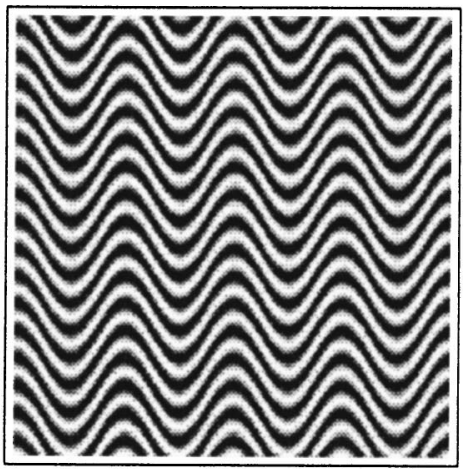

(f)

Fig. 1. Various curvilinear gratings $r(x, y)$ that have a periodic profile waveform of $\cos \left(2 \pi f x^{\prime}\right)$ : (a) straight cosinusoidal grating: $\cos (2 \pi f x)$, (b) rotated straight cosinusoidal grating: $\cos \{2 \pi f[x \cos \theta+y \sin \theta]\}$, (c) parabolic $\operatorname{cosinusoidal~grating:~} \cos \{2 \pi f[y$ $\left.\left.-0.15 x^{2}\right]\right\}$, (d) circular cosinusoidal grating: $\cos \left(2 \pi f \sqrt{x^{2}+y^{2}}\right), \quad(\mathrm{e}) \arg \sinh (x)$-shaped cosinusoidal grating: $\cos \{2 \pi f[y$ - $\arg \sinh (x)]$, (f) cosinusoidally shaped grating: $\cos \{2 \pi f[y-\cos (2 \pi f x / 4)]\}$. 


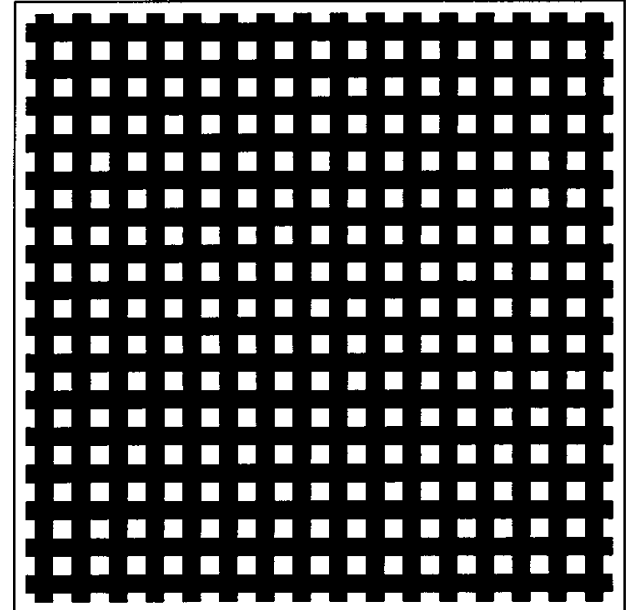

(a)

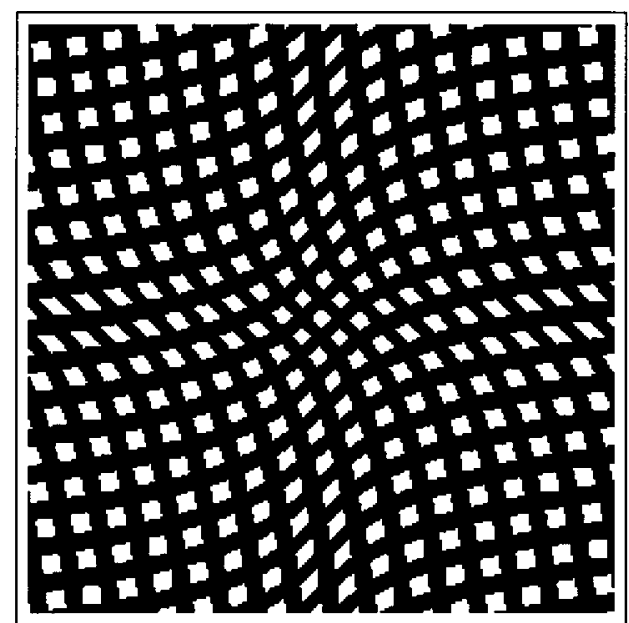

(b)

Fig. 2. (a) Periodic binary line grid $p\left(x^{\prime}, y^{\prime}\right)$ of example 3, (b) curved binary grid $r(x, y)$ obtained by applying on $p\left(x^{\prime}, y^{\prime}\right)$ the $2 \mathrm{D}$ nonlinear transformation $\mathbf{g}(x, y)=[-x-\arg \sinh (y), y-\arg \sinh (x)]$. Note that both line grids can be seen also as dot screens of white dots on a black background.

1(a)] and that we bend its parallel straight corrugations into parallel parabolas of the shape $y=a x^{2}+c$ without changing their cosinusoidal profile form [see Fig. 1(c)]. This can be described mathematically as a nonlinear transformation $x^{\prime}=g_{1}(x, y)=y-a x^{2}$, where $a$ is a nonzero constant that defines the bending rate of the parabolas. $^{12}$ (Note that the level lines $x^{\prime}=n$ of the surface $x^{\prime}=y-a x^{2}$ over the $x, y$ plane are indeed the required parabolas $y=a x^{2}+n$.) The parabolic cosinusoidal grating obtained by applying this bending transformation on the original grating $p\left(x^{\prime}\right)$ is given, therefore, by $r(x, y)=p\left(y-a x^{2}\right)=\cos \left[2 \pi f\left(y-a x^{2}\right)\right]$. Its geometric layout is given by the locus of its maxima in the $x, y$ plane, namely, $2 \pi f\left(y-a x^{2}\right)=2 \pi k, k \in \mathbb{Z}$, and its normalized periodic profile is $\cos \left(2 \pi x^{\prime}\right)$.

Example 2: In the case of a circular grating with a cosinusoidal periodic profile [Fig. 1(d)], the original, uncurved periodic wave (the periodic profile of the circular grating) is again, this time using the letter $r^{\prime}$ rather than $x^{\prime}, p\left(r^{\prime}\right)=\cos \left(2 \pi f r^{\prime}\right)$. The circular grating $r(x, y)$ is obtained from $p\left(r^{\prime}\right)$ by replacing $r^{\prime}$ with $\sqrt{x^{2}+y^{2}}$ :

$$
r(x, y)=p\left(\sqrt{x^{2}+y^{2}}\right)=\cos \left(2 \pi f \sqrt{x^{2}+y^{2}}\right) .
$$

Let us proceed now to the case of curved dot screens (or line grids). Assume that the curved dot screen $r(x, y)$ is obtained by bending a two-fold periodic dot screen $p\left(x^{\prime}, y^{\prime}\right)$, i.e., by replacing $x^{\prime}$ and $y^{\prime}$ with the functions $x^{\prime}=g_{1}(x, y)$ and $y^{\prime}=g_{2}(x, y)$, respectively: $r(x, y)$ $=p\left[g_{1}(x, y), g_{2}(x, y)\right]$. An example of such a curved dot screen $r(x, y)$ is shown in Fig. 2(b). The intensity profile of the original, uncurved two-fold periodic screen $p\left(x^{\prime}, y^{\prime}\right)$ is called the periodic profile of the curved screen $r(x, y)$. The periodic profile of a curved screen may be any two-fold periodic waveform; as in the case of curvilinear gratings, we will use the term normalized periodic profile whenever we choose $p\left(x^{\prime}, y^{\prime}\right)$ to have a unit frequency (to both directions). The functions $x^{\prime}=g_{1}(x, y)$ and $y^{\prime}=g_{2}(x, y)$ that bend $p\left(x^{\prime}, y^{\prime}\right)$ into the curved screen $r(x, y)$ are called the bending transformation.
A curved screen $r(x, y)=p\left[g_{1}(x, y), g_{2}(x, y)\right]$ is therefore characterized by two basic and independent properties: its geometric layout, which is determined by the functions $g_{1}(x, y)$ and $g_{2}(x, y)$, and its intensity behavior within each "curved period," which is determined by the two-fold periodic profile $p\left(x^{\prime}, y^{\prime}\right)$.

In fact, this bending process can be interpreted as a mapping of $\mathbb{R}^{2}$ onto itself, or, equivalently, as a coordinate change in $\mathbb{R}^{2}$ from the original $x^{\prime}, y^{\prime}$ coordinate system into the $x, y$ system. ${ }^{13}$ This $2 \mathrm{D}$ coordinate transformation is specified for each of the two original directions separately by the bending functions $x^{\prime}=g_{1}(x, y)$ and $y^{\prime}=g_{2}(x, y)$, which transform the new $x, y$ coordinates back into the original $x^{\prime}, y^{\prime}$ coordinates. The effect of this $2 \mathrm{D}$ coordinate transformation can be expressed, then, by

$$
\mathbf{g :}\left(\begin{array}{l}
x \\
y
\end{array}\right) \rightarrow\left(\begin{array}{l}
x^{\prime} \\
y^{\prime}
\end{array}\right),
$$

where

$$
\left(\begin{array}{l}
x^{\prime} \\
y^{\prime}
\end{array}\right)=\left(\begin{array}{l}
g_{1}(x, y) \\
g_{2}(x, y)
\end{array}\right),
$$

or in a more compact vector notation: $\mathbf{x}^{\prime}=\mathbf{g}(\mathbf{x})$. Note that $\mathbf{g}(\mathbf{x})$ is a mapping of $R^{2}$ onto itself: $\mathbf{g}: R^{2} \rightarrow R^{2}$; we denote it by a boldface letter $\mathbf{g}$ since the value it returns, $\mathbf{g}(\mathbf{x})$, is a vector. Clearly, in order that the image of this mapping span the whole $x, y$ plane $\mathrm{R}^{2}$, the two individual coordinate transformations $x^{\prime}=g_{1}(x, y)$ and $y^{\prime}=g_{2}(x, y)$ must be independent; i.e., there must exist no function $f\left(x^{\prime}, y^{\prime}\right)$ such that $f\left[g_{1}(x, y), g_{2}(x, y)\right]=0$ is satisfied for all $(x, y){ }^{14}$ Equivalently, this means that the Jacobian

$$
J(x, y)=\left|\begin{array}{ll}
\frac{\partial g_{1}}{\partial x} & \frac{\partial g_{1}}{\partial y} \\
\frac{\partial g_{2}}{\partial x} & \frac{\partial g_{2}}{\partial y}
\end{array}\right|
$$


is not identically zero. ${ }^{15}$ In order to avoid unnecessary mathematic complications, we will generally assume that the bending transformation $\mathbf{g}(\mathbf{x})$ is a diffeomorphism on $\mathbb{R}^{2}$, i.e., a one-to-one continuously differentiable mapping of $R^{2}$ onto itself whose inverse mapping is also continuously differentiable. This ensures that the bending transformation has no abrupt jumps or other troublesome singularities.

Example 3: Assume that we are given a periodic binary line grid $p\left(x^{\prime}, y^{\prime}\right)$ that is the superposition of a vertical square-wave grating $p_{1}\left(x^{\prime}, y^{\prime}\right)=\sum_{m=-\infty}^{\infty} \operatorname{rect}\left[\left(x^{\prime}\right.\right.$ - $m T) / \tau]$ and a horizontal square-wave grating $p_{2}\left(x^{\prime}, y^{\prime}\right)=\sum_{n=-\infty}^{\infty} \operatorname{rect}\left[\left(y^{\prime}-n T\right) / \tau\right]$, both having the same period $T$ and the same opening $\tau$; that is, $p\left(x^{\prime}, y^{\prime}\right)=p_{1}\left(x^{\prime}, y^{\prime}\right) p_{2}\left(x^{\prime}, y^{\prime}\right)=\Sigma_{m=-\infty}^{\infty} \Sigma_{n=-\infty}^{\infty} \operatorname{rect}\left[\left(x^{\prime}\right.\right.$ - $m T) / \tau] \operatorname{rect}\left[\left(y^{\prime}-n T\right) / \tau\right]$. We define the 2D nonlinear transformation $\mathbf{g}(x, y)$ as follows:

$$
\left(\begin{array}{l}
x^{\prime} \\
y^{\prime}
\end{array}\right)=\mathbf{g}\left(\begin{array}{l}
x \\
y
\end{array}\right)=\left(\begin{array}{l}
g_{1}(x, y) \\
g_{2}(x, y)
\end{array}\right)=\left(\begin{array}{c}
-x-\arg \sinh (y) \\
y-\arg \sinh (x)
\end{array}\right) .
$$

By applying the nonlinear transformation $\mathbf{g}(x, y)$ on the periodic binary line grid $p\left(x^{\prime}, y^{\prime}\right)$, we obtain the curved binary grid $r(x, y)$, as shown in Fig. 2:

$$
\begin{aligned}
r(x, y)= & p[-x-\arg \sinh (y), y-\arg \sinh (x)] \\
= & \sum_{m=-\infty}^{\infty} \sum_{n=-\infty}^{\infty} \operatorname{rect}\left[\frac{-x-\arg \sinh (y)-m T}{\tau}\right] \\
& \times \operatorname{rect}\left[\frac{y-\arg \sinh (x)-n T}{\tau}\right] .
\end{aligned}
$$

\section{FOURIER DECOMPOSITION OF REPETITIVE STRUCTURES}

Our next step in the analysis of repetitive structures with a given periodic profile is based on the Fourier-series development of their periodic profiles. We start, again, with the case of curvilinear gratings. Assume that the curvilinear grating $r(x, y)$ is obtained by bending a periodic grating $p\left(x^{\prime}\right)$, i.e., by replacing $x^{\prime}$ with a function $x^{\prime}=g_{1}(x, y): \quad r(x, y)=p\left[g_{1}(x, y)\right] . \quad$ A few examples of curvilinear gratings $r(x, y)$ with a square-wave periodic-profile form $p\left(x^{\prime}\right)$ are shown in Fig. 3. We first consider the Fourier development of the original grating $p\left(x^{\prime}\right)$ :

$$
p\left(x^{\prime}\right)=\sum_{n=-\infty}^{\infty} c_{n} \exp \left(i 2 \pi n f x^{\prime}\right)
$$

Then we replace $x^{\prime}$ in this Fourier series with the function $g_{1}(x, y)$, which defines the curvilinear behavior of the grating $r(x, y)$ throughout the plane, keeping the same coefficients $c_{n}$ as in the Fourier decomposition of $p\left(x^{\prime}\right)$ :

$$
r(x, y)=p\left[g_{1}(x, y)\right]=\sum_{n=-\infty}^{\infty} c_{n} \exp \left[i 2 \pi n f g_{1}(x, y)\right] .
$$

This is therefore the Fourier decomposition of our curvilinear grating $r(x, y)$. This approach has been introduced in Ref. 16, where the periodic profile $p\left(x^{\prime}\right)$ was taken as a binary square wave.

As we have already seen, this change of variables is in fact equivalent to a coordinate change in the image domain [for example, in the case of Fig. 3(b), from polar to Cartesian coordinates].

We proceed now to the 2D case of a curved dot screen (or a curved line grid). Assume that the curved dot screen $r(x, y)$ is obtained by bending a $2 \mathrm{D}$ periodic dot screen $p\left(x^{\prime}, y^{\prime}\right)$, i.e., by replacing $x^{\prime}$ and $y^{\prime}$ with functions $x^{\prime}=g_{1}(x, y)$ and $y^{\prime}=g_{2}(x, y): r(x, y)=$ $p\left[g_{1}(x, y), g_{2}(x, y)\right]$. An example of such a curved dot screen $r(x, y)$ is shown in Fig. 2(b). According to the present approach, we first consider the Fourier development of the original $2 \mathrm{D}$ periodic dot screen $p\left(x^{\prime}, y^{\prime}\right)$ :

$$
\begin{aligned}
p\left(x^{\prime}, y^{\prime}\right)= & \sum_{m=-\infty}^{\infty} \sum_{n=-\infty}^{\infty} c_{m, n} \\
& \times \exp \left[i 2 \pi\left(m x^{\prime} / T_{x^{\prime}}+n y^{\prime} / T_{y^{\prime}}\right)\right] .
\end{aligned}
$$

Then we replace $x^{\prime}$ and $y^{\prime}$ in this Fourier series with the functions $g_{1}(x, y)$ and $g_{2}(x, y)$, which define the curved behavior of the grating $r(x, y)$ throughout the plane, keeping the same coefficients $c_{m, n}$ as in the Fourier decomposition of $p\left(x^{\prime}, y^{\prime}\right)$ :

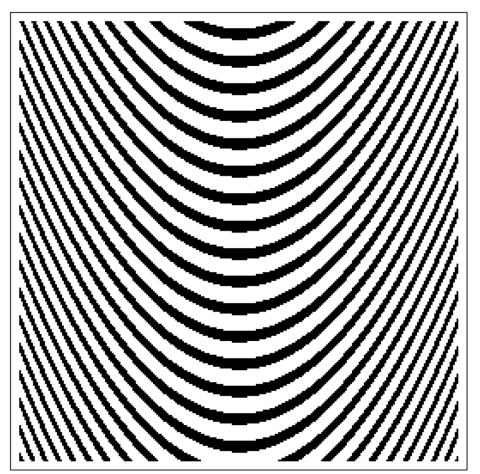

(a)

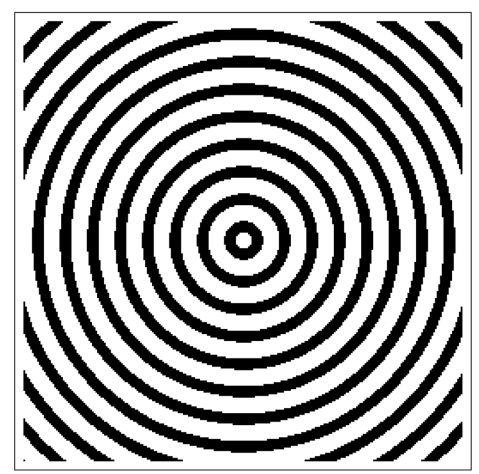

(b)

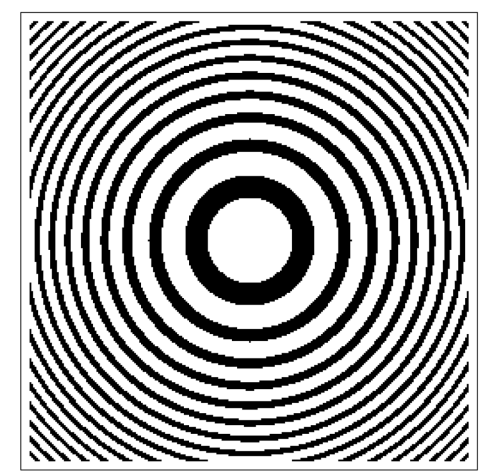

(c)

Fig. 3. Some examples of curvilinear gratings $r(x, y)$ having a square-wave periodic profile (with opening ratio $\tau / T=0.6$ ) and a bending transformation $g_{1}(x, y)$. (a) Parabolic grating: $g_{1}(x, y)=y-0.15 x^{2}$, (b) circular grating: $g_{1}(x, y)=\sqrt{x^{2}+y^{2}}$, (c) circular zone grating: $g_{1}(x, y)=\left(x^{2}+y^{2}\right) / 8$. 


$$
\begin{aligned}
r(x, y)= & p\left[g_{1}(x, y), g_{2}(x, y)\right] \\
= & \sum_{m=-\infty}^{\infty} \sum_{n=-\infty}^{\infty} c_{m, n} \exp \left\{i 2 \pi \left[m g_{1}(x, y) / T_{x^{\prime}}\right.\right. \\
& \left.\left.+n g_{2}(x, y) / T_{y^{\prime}}\right]\right\} .
\end{aligned}
$$

This is therefore the Fourier decomposition of our curved dot screen $r(x, y)$.

Remark: We will usually prefer to choose $p\left(x^{\prime}, y^{\prime}\right)$ as a normalized periodic profile with $T_{x^{\prime}}=1, T_{y^{\prime}}=1$; and if $T_{x^{\prime}} \neq 1$ or $T_{y^{\prime}} \neq 1$, we will consider them to be included within the functions $g_{1}(x, y), g_{2}(x, y)$, leaving $p\left(x^{\prime}, y^{\prime}\right)$ itself normalized. Therefore $T_{x^{\prime}}$ and $T_{y^{\prime}}$ will usually be omitted from Eq. (4).

Equation (2) or its 2D counterpart, Eq. (4), are simply a formal Fourier decomposition of the curved layer $r(x, y)$ into a sum of curvilinear exponentials [or cosines, if $p\left(x^{\prime}, y^{\prime}\right)$ is symmetric], which were all subjected to the same coordinate transformation $\mathbf{g}(x, y)$ as $r(x, y)$ itself. This decomposition is simply an alternative representation of the curvilinear layer $r(x, y)$ in the image domain.

\section{SUPERPOSITION MOIRÉS BETWEEN REPETITIVE LAYERS}

Let us start once again with the simpler case of curvilinear gratings. Suppose that the original repetitive layers are given by the curvilinear gratings

$$
\begin{aligned}
& r_{1}(x, y)=\sum_{m=-\infty}^{\infty} c_{m}^{(1)} \exp \left[i 2 \pi m g_{1}(x, y)\right], \\
& r_{2}(x, y)=\sum_{n=-\infty}^{\infty} c_{n}^{(2)} \exp \left[i 2 \pi n g_{2}(x, y)\right] .
\end{aligned}
$$

Their superposition is expressed therefore by the product

$$
\begin{aligned}
r_{1}(x, y) r_{2}(x, y)= & \left\{\sum_{m=-\infty}^{\infty} c_{m}^{(1)} \exp \left[i 2 \pi m g_{1}(x, y)\right]\right\} \\
& \times\left\{\sum_{n=-\infty}^{\infty} c_{n}^{(2)} \exp \left[i 2 \pi n g_{2}(x, y)\right]\right\} \\
= & \sum_{m=-\infty}^{\infty} \sum_{n=-\infty}^{\infty} c_{m}^{(1)} c_{n}^{(2)} \exp \left\{i 2 \pi \left[m g_{1}(x, y)\right.\right. \\
& \left.\left.+n g_{2}(x, y)\right]\right\} .
\end{aligned}
$$

We call the term in this double sum whose indices are $m$ and $n$ the $(m, n)$ term.

Consider now the partial sum that consists of all the terms of this double sum that are spanned by the $\left(k_{1}, k_{2}\right)$ term. This partial sum consists of all the $m\left(k_{1}, k_{2}\right)$ terms $(m \in \mathbb{Z})$, i.e., all the terms whose indices are $m k_{1}$ and $m k_{2}$ :

$$
\begin{aligned}
m_{k_{1}, k_{2}}(x, y)= & \sum_{m=-\infty}^{\infty} c_{m k_{1}}^{(1)} c_{m k_{2}}^{(2)} \\
& \times \exp \left\{i 2 \pi m\left[k_{1} g_{1}(x, y)+k_{2} g_{2}(x, y)\right]\right\} .
\end{aligned}
$$

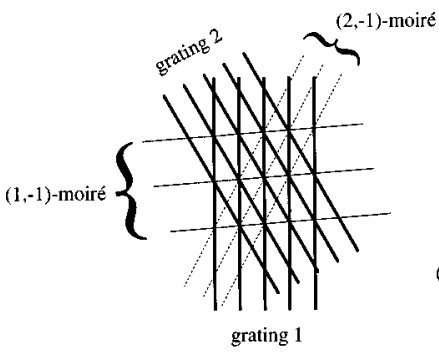

(a)

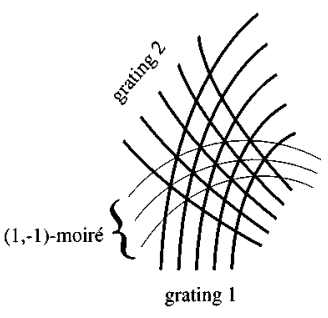

(b)
Fig. 4. Schematic illustration of a $(1,-1)$ moiré (a) between two straight periodic gratings, (b) between two curvilinear gratings. The dotted lines in (a), which represent the $(2,-1)$ moiré, have been omitted in (b) for the sake of clarity.

This partial sum corresponds to a repetitive structure that is present in the superposition $r_{1}(x, y) r_{2}(x, y)$ but is not present in either of the original layers $r_{1}(x, y)$ and $r_{2}(x, y)$ themselves. This structure is called the $\left(k_{1}, k_{2}\right)$ moiré; this moiré becomes visible in the superposition whenever its amplitude is sufficiently strong and its frequency is smaller than the maximum frequency that can be resolved by the eye at the corresponding viewing conditions.

As an example, the $(1,-1)$ moiré is defined by the partial sum consisting of all the terms of the double sum [Eq. (5)] whose indices are $m$ and $-m$, namely,

$$
\begin{aligned}
m_{1,-1}(x, y)= & \sum_{m=-\infty}^{\infty} c_{m}^{(1)} c_{-m}^{(2)} \\
& \times \exp \left\{i 2 \pi m\left[g_{1}(x, y)-g_{2}(x, y)\right]\right\} .
\end{aligned}
$$

Figure 4 schematically shows the $(1,-1)$ moiré between two straight gratings and between two curvilinear gratings. Other examples of moiré between periodic layers have been presented in Ref. 10 .

Note that in the case where $g_{1}(x, y)$ and $g_{2}(x, y)$ are linear functions of $x$ and $y, g_{1}(x, y)=u_{1} x+\nu_{1} y$, $g_{2}(x, y)=u_{2} x+\nu_{2} y$, the layers $r_{1}(x, y)$ and $r_{2}(x, y)$ are periodic, and their frequencies are given, respectively, by the vectors $\mathbf{f}_{1}=\left(u_{1}, \nu_{1}\right)$ and $\mathbf{f}_{2}=\left(u_{2}, \nu_{2}\right)$ in the spectrum. The $\left(k_{1}, k_{2}\right)$ moire [Eq. (6)] is, in this case, a periodic function whose frequency is $k_{1} \mathbf{f}_{1}+k_{2} \mathbf{f}_{2}$. For example, the $(1,-1)$ moire has the frequency $\mathbf{f}_{1}-\mathbf{f}_{2}$, the $(1,1)$ moiré has the frequency $\mathbf{f}_{1}+\mathbf{f}_{2}$, the $(2,-1)$ moiré has the frequency $2 \mathbf{f}_{1}-\mathbf{f}_{2}$, etc. These moirés correspond, indeed, to the difference beat, the sum beat, and the various higher-order beats that are generated in the superposition, and Eq. (6) gives their Fourier-series decomposition. As we can see, our systematic formalism for denoting the moiré effects indeed covers all possible cases.

Now all the substructures of the superposition that are defined by the $\left(k_{1}, k_{2}\right)$ moirés exist in the superposition simultaneously (they are, in fact, partial sums of the Fourier-series decomposition of the superposition). However, depending on whether their frequency vectors $k_{1} \mathbf{f}_{1}$ $+k_{2} \mathbf{f}_{2}$ fall in the spectrum inside or outside the range of visible frequencies, any of these moirés may be visible or 
invisible. For example, if the difference frequency $\mathbf{f}_{1}-\mathbf{f}_{2}$ falls inside the visible frequency range but the sum frequency $\mathbf{f}_{1}+\mathbf{f}_{2}$ falls beyond the visible frequency range, then the $(1,-1)$ moire (the difference beat) is visible in the superposition but the $(1,1)$ moire (the sum beat) is not visible. This has been explained in detail in Ref. 10. In the case of curved gratings, in which the frequency vector of each layer is no longer constant but rather varies throughout the $x, y$ plane, we consider at any point $(x, y)$ the local frequency vectors $\mathbf{f}_{1}(x, y)$, $\mathbf{f}_{2}(x, y)$, etc. The same visibility rule applies here as above-but this time it applies locally, i.e., at any point $(x, y)$ of the superposition individually. Therefore in the case of curved layers any of the moiré effects may be visible in some zones of the $x, y$ plane and invisible in other zones.

Proceeding now to the superposition of curved dot screens, suppose that the original curved layers $r_{1}(x, y)$ and $r_{2}(x, y)$ are given by the curved dot screens

$$
\begin{aligned}
r_{1}(x, y)= & \sum_{m=-\infty}^{\infty} \sum_{n=-\infty}^{\infty} c_{m, n}^{(1)} \\
& \times \exp \left\{i 2 \pi\left[m g_{1}(x, y)+n g_{2}(x, y)\right]\right\}, \\
r_{2}(x, y)= & \sum_{m=-\infty}^{\infty} \sum_{n=-\infty}^{\infty} c_{m, n}^{(2)} \\
& \times \exp \left\{i 2 \pi\left[m g_{3}(x, y)+n g_{4}(x, y)\right]\right\} .
\end{aligned}
$$

Their superposition is expressed therefore by the product

$$
\begin{aligned}
& r_{1}(x, y) r_{2}(x, y) \\
& =\left(\sum_{m=-\infty}^{\infty} \sum_{n=-\infty}^{\infty} c_{m, n}^{(1)} \exp \left\{i 2 \pi\left[m g_{1}(x, y)+n g_{2}(x, y)\right]\right\}\right) \\
& \quad \times\left(\sum_{m=-\infty}^{\infty} \sum_{n=-\infty}^{\infty} c_{m, n}^{(2)} \exp \left\{i 2 \pi\left[m g_{3}(x, y)+n g_{4}(x, y)\right]\right\}\right) \\
& =\sum_{n_{1}=-\infty} \sum_{n_{2}=-\infty} \sum_{n_{3}=-\infty}^{\infty} \sum_{n_{4}=-\infty}^{\infty} c_{n_{1}, n_{2}}^{(1)} c_{n_{3}, n_{4}}^{(2)} \\
& \quad \times \exp \left\{i 2 \pi \left[n_{1} g_{1}(x, y)+n_{2} g_{2}(x, y)+n_{3} g_{3}(x, y)\right.\right. \\
& \left.\left.\quad+n_{4} g_{4}(x, y)\right]\right\} .
\end{aligned}
$$

We call the term in this sum whose indices are $n_{1}, n_{2}$, $n_{3}$, and $n_{4}$ the $\left(n_{1}, n_{2}, n_{3}, n_{4}\right)$ term.

Consider the 2D partial sum that consists of all the terms of this quadruple sum that are spanned by the $\left(k_{1}, k_{2}, k_{3}, k_{4}\right)$ term and its orthogonal counterpart, the $\left(-k_{2}, k_{1},-k_{4}, k_{3}\right)$ term [note that the indices $\left(k_{1}, k_{2}, k_{3}, k_{4}\right)$ and $\left(-k_{2}, k_{1},-k_{4}, k_{3}\right)$ are orthogonal since their inner product is zero]. This $2 \mathrm{D}$ partial sum consists of all the terms whose indices are $m k_{1}-n k_{2}$, $m k_{2}+n k_{1}, m k_{3}-n k_{4}$, and $m k_{4}+n k_{3}$, since

$$
\begin{aligned}
& m\left(k_{1}, k_{2}, k_{3}, k_{4}\right)+n\left(-k_{2}, k_{1},-k_{4}, k_{3}\right) \\
& =\left(m k_{1}-n k_{2}, m k_{2}+n k_{1}, m k_{3}-n k_{4}, m k_{4}+n k_{3}\right),
\end{aligned}
$$

and it is therefore given by

$$
\begin{aligned}
& m_{k_{1}, k_{2}, k_{3}, k_{4}}(x, y) \\
& =\sum_{m=-\infty}^{\infty} \sum_{n=-\infty}^{\infty} c_{m k_{1}-n k_{2}, m k_{2}+n k_{1}}^{(1)} c_{m k_{3}}^{(2)}-n k_{4}, m k_{4}+n k_{3} \\
& \quad \times \exp \left\{i 2 \pi \left[\left(m k_{1}-n k_{2}\right) g_{1}(x, y)\right.\right. \\
& \quad+\left(m k_{2}+n k_{1}\right) g_{2}(x, y)+\left(m k_{3}-n k_{4}\right) g_{3}(x, y) \\
& \left.\left.\quad+\left(m k_{4}-n k_{3}\right) g_{4}(x, y)\right]\right\} \\
& =\sum_{m=-\infty}^{\infty} \sum_{n=-\infty}^{\infty} c_{m k_{1}-n k_{2}, m k_{2}+n k_{1}}^{(1)} c_{m k_{3}-n k_{4}, m k_{4}+n k_{3}}^{(2)} \\
& \quad \times \exp \left(i 2 \pi \left\{m \left[k_{1} g_{1}(x, y)+k_{2} g_{2}(x, y)+k_{3} g_{3}(x, y)\right.\right.\right. \\
& \left.\quad+k_{4} g_{4}(x, y)\right]+n\left[-k_{2} g_{1}(x, y)\right. \\
& \left.\left.\left.\quad+k_{1} g_{2}(x, y)-k_{4} g_{3}(x, y)+k_{3} g_{4}(x, y)\right]\right\}\right) .
\end{aligned}
$$

This partial sum corresponds to a $2 \mathrm{D}$ repetitive structure that is present in the superposition $r_{1}(x, y) r_{2}(x, y)$ but is not present in either of the original layers $r_{1}(x, y)$ and $r_{2}(x, y)$ themselves. This $2 \mathrm{D}$ structure is called the $\left(k_{1}, k_{2}, k_{3}, k_{4}\right)$ moiré.

As an example, the $(1,0,-1,0)$ moire is defined by the partial sum consisting of all the terms of the quadruple sum [Eq. (8)] whose indices are $m, n,-m,-n$, namely,

$$
\begin{aligned}
m_{1,0,-1,0}(x, y) & \\
= & \sum_{m=-\infty}^{\infty} \sum_{n=-\infty}^{\infty} c_{m, n}^{(1)} c_{-m,-n}^{(2)} \exp \left\{i 2 \pi \left[m g_{1}(x, y)\right.\right. \\
& \left.\left.+n g_{2}(x, y)-m g_{3}(x, y)-n g_{4}(x, y)\right]\right\} \\
= & \sum_{m=-\infty}^{\infty} \sum_{n=-\infty}^{\infty} c_{m, n}^{(1)} c_{-m,-n}^{(2)} \exp \left(i 2 \pi \left\{m \left[g_{1}(x, y)\right.\right.\right. \\
& \left.\left.\left.-g_{3}(x, y)\right]+n\left[g_{2}(x, y)-g_{4}(x, y)\right]\right\}\right) .
\end{aligned}
$$

This is indeed the $2 \mathrm{D}$ equivalent of the difference beat between two gratings, the $(1,-1)$ moiré [see Eq. (7)].

Actual examples of such moirés are given in the next section. Note that the above definitions of the $\left(k_{1}, k_{2}\right)$ moiré between two curvilinear gratings or the $\left(k_{1}, k_{2}, k_{3}, k_{4}\right)$ moiré between two curved dot screens are in fact generalizations of the respective definitions in the periodic case: In the periodic case each term in the sums (5) or (8) corresponds to an impulse in the spectrum of the superposition, and each moiré effect is represented by a partial cluster of impulses in that spectrum (see Ref. 10).

\section{MOIRÉ EXTRACTION IN THE SUPERPOSITION OF REPETITIVE LAYERS}

A. Moiré Extraction in the Superposition of Curvilinear Gratings

We arrive now at the main part of our paper. We start by presenting the $T$-convolution theorem in terms of Fourier series ${ }^{17}$ :

T-convolution theorem: Let $p_{1}(x)$ and $p_{2}(x)$ be periodic functions of the period $T$ integrable on a one-period interval $(0, T)$, and let their Fourier series representations be 


$$
\begin{aligned}
& p_{1}(x)=\sum_{m=-\infty}^{\infty} c_{m}^{(1)} \exp (i 2 \pi m x / T) \\
& p_{2}(x)=\sum_{n=-\infty}^{\infty} c_{n}^{(2)} \exp (i 2 \pi n x / T) .
\end{aligned}
$$

Then their $T$ convolution

$$
h(x)=p_{1}(x) * p_{2}(x)=\frac{1}{T} \int_{T} p_{1}\left(x-x^{\prime}\right) p_{2}\left(x^{\prime}\right) \mathrm{d} x^{\prime}
$$

is also periodic with the same period $T$, and its Fourier series representation is

$$
h(x)=\sum_{m=-\infty}^{\infty} c_{m}^{(1)} c_{m}^{(2)} \exp (i 2 \pi m x / T) .
$$

Now let $r_{1}(x, y)$ and $r_{2}(x, y)$ be two curvilinear gratings given by

$$
\begin{aligned}
& r_{1}(x, y)=p_{1}\left[g_{1}(x, y)\right]=\sum_{m=-\infty}^{\infty} c_{m}^{(1)} \exp \left[i 2 \pi m g_{1}(x, y)\right], \\
& r_{2}(x, y)=p_{2}\left[g_{2}(x, y)\right]=\sum_{n=-\infty}^{\infty} c_{n}^{(2)} \exp \left[i 2 \pi n g_{2}(x, y)\right],
\end{aligned}
$$

where $\quad p_{1}\left(x^{\prime}\right)=\Sigma_{m=-\infty}^{\infty} c_{m}^{(1)} \exp \left(i 2 \pi m x^{\prime}\right)$ and $p_{2}\left(x^{\prime}\right)$ $=\sum_{n=-\infty}^{\infty} c_{n}^{(2)} \exp \left(i 2 \pi n x^{\prime}\right)$ are the onefold periodic profiles of the gratings $r_{1}(x, y)$ and $r_{2}(x, y)$, normalized to the period $\mathbf{T}=(1,0)$, and $g_{1}(x, y)$ and $g_{2}(x, y)$ are the coordinate transformations that transform $p_{1}\left(x^{\prime}\right)$ and $p_{2}\left(x^{\prime}\right)$ into the gratings $r_{1}(x, y)$ and $r_{2}(x, y)$, respectively.

As we have seen in Section 4 [see Eq. (5)], the superposition of the two gratings is expressed by the product

$$
\begin{aligned}
r_{1}(x, y) r_{2}(x, y)= & \sum_{m=-\infty}^{\infty} \sum_{n=-\infty}^{\infty} c_{m}^{(1)} c_{n}^{(2)} \\
& \times \exp \left\{i 2 \pi\left[m g_{1}(x, y)+n g_{2}(x, y)\right]\right\},
\end{aligned}
$$

and the partial sum that corresponds to the $(1,-1)$ moiré consists of all the terms of this double sum whose indices are $m$ and $-m$, namely [see Eq. (7)],

$$
\begin{aligned}
m_{1,-1}(x, y)= & \sum_{m=-\infty}^{\infty} c_{m}^{(1)} c_{-m}^{(2)} \\
& \times \exp \left\{i 2 \pi m\left[g_{1}(x, y)-g_{2}(x, y)\right]\right\} .
\end{aligned}
$$

Let us denote by $p_{1,-1}\left(x^{\prime}\right)$ the periodic profile [normalized to the same period $\mathbf{T}=(1,0)]$ of $m_{1,-1}(x, y)$ :

$$
p_{1,-1}\left(x^{\prime}\right)=\sum_{m=-\infty}^{\infty} c_{m}^{(1)} c_{-m}^{(2)} \exp \left(i 2 \pi m x^{\prime}\right) .
$$

We see, therefore, by the $T$-convolution theorem that $p_{1,-1}\left(x^{\prime}\right)$ is simply the $T$ convolution

$$
p_{1,-1}\left(x^{\prime}\right)=p_{1}\left(x^{\prime}\right) * p_{2}\left(-x^{\prime}\right),
$$

where $x^{\prime}=g_{1}(x, y)-g_{2}(x, y)$ is the coordinate transformation that brings $p_{1,-1}\left(x^{\prime}\right)$ into the geometric layout of the $(1,-1)$ moiré. In other words, we obtain the following:
Result 1: The $(1,-1)$ moiré $m_{1,-1}(\mathbf{x})$ in the superposition of two curvilinear gratings $r_{1}(\mathbf{x})=p_{1}\left[g_{1}(\mathbf{x})\right]$ and $r_{2}(\mathbf{x})=p_{2}\left[g_{2}(\mathbf{x})\right] \quad$ is given by $m_{1,-1}(\mathbf{x})$ $=p_{1,-1}\left[g_{1,-1}(\mathbf{x})\right]$, where

1. $p_{1,-1}(\mathbf{x})$, the normalized periodic profile of the $(1,-1)$ moire, is the $T$ convolution of the normalized periodic profiles of the original gratings:

$$
p_{1,-1}\left(x^{\prime}\right)=p_{1}\left(x^{\prime}\right) * p_{2}\left(-x^{\prime}\right) ;
$$

2. $g_{1,-1}(\mathbf{x})$, the coordinate transformation that brings $p_{1,-1}\left(x^{\prime}\right)$ into the $(1,-1)$ moiré $m_{1,-1}(\mathbf{x})$, is given by

$$
g_{1,-1}(\mathbf{x})=g_{1}(\mathbf{x})-g_{2}(\mathbf{x}) .
$$

Using less formal language, we may formulate this result as follows:

Result 2: The $(1,-1)$ moiré $m_{1,-1}(x, y)$ generated in the superposition of the two curvilinear gratings $r_{1}(x, y)=p_{1}\left[g_{1}(x, y)\right]$ and $r_{2}(x, y)=p_{2}\left[g_{2}(x, y)\right]$ can be seen as the result of a three-stage process:

1. Normalization of the original curvilinear gratings by replacing in each of them $g_{i}(x, y)$ with $x^{\prime}$ [i.e., by applying to each of them the inverse coordinate transformation $\left.g_{i}(x, y) \rightarrow x^{\prime}\right]$, in order to straighten them into uncurved, normalized periodic gratings that have identical periods $\mathbf{T}=(1,0)$.

2. $T$ convolution of these normalized periodic line gratings. This gives the uncurved, normalized periodic profile of the $(1,-1)$ moiré, with the same period $\mathbf{T}$ $=(1,0)$.

3. Bending this normalized periodic profile of the moiré into the actual curvilinear geometric layout of the moiré, by replacing $x^{\prime}$ with $g_{1}(x, y)-g_{2}(x, y)$ (i.e., by applying the nonlinear coordinate transformation $x^{\prime}$ $\rightarrow g_{1}\left[(x, y)-g_{2}(x, y)\right]$.

More generally, we have seen in Section 4 that the partial sum of Eq. (11) that corresponds to the $\left(k_{1}, k_{2}\right)$ moiré consists of all the terms of the double sum whose indices are $m k_{1}$ and $m k_{2}$, namely [see Eq. (6)],

$$
\begin{aligned}
m_{k_{1}, k_{2}}(x, y)= & \sum_{m=-\infty}^{\infty} c_{m k_{1}}^{(1)} c_{m k_{2}}^{(2)} \\
& \times \exp \left\{i 2 \pi m\left[k_{1} g_{1}(x, y)+k_{2} g_{2}(x, y)\right]\right\} .
\end{aligned}
$$

If we denote by $p_{k_{1}, k_{2}}\left(x^{\prime}\right)$ the periodic profile of $m_{k_{1}, k_{2}}(x, y)$ [normalized to the period $\mathbf{T}=(1,0)$ ],

$$
p_{k_{1}, k_{2}}\left(x^{\prime}\right)=\sum_{m=-\infty}^{\infty} c_{m k_{1}}^{(1)} c_{m k_{2}}^{(2)} \exp \left(i 2 \pi m x^{\prime}\right),
$$

then we see by the $T$-convolution theorem that $p_{k_{1}, k_{2}}\left(x^{\prime}\right)$ is simply the $T$ convolution,

$$
p_{k_{1}, k_{2}}\left(x^{\prime}\right)=\operatorname{sub}_{k_{1}}\left[p_{1}\left(x^{\prime}\right)\right] * \operatorname{sub}_{k_{2}}\left[p_{2}\left(x^{\prime}\right)\right],
$$

where $\operatorname{sub}_{k}\left[p\left(x^{\prime}\right)\right]$ is the $k$-sub-Fourier series of $p\left(x^{\prime}\right)$, i.e., the periodic function (with period $\mathbf{T}=(1,0)$ ) whose Fourier series contains only every $k$ th coefficient from the Fourier series $p\left(x^{\prime}\right)=\sum_{m=-\infty}^{\infty} c_{m} \exp \left(i 2 \pi m x^{\prime}\right)$ :

$$
\operatorname{sub}_{k}\left[p\left(x^{\prime}\right)\right]=\sum_{m=-\infty}^{\infty} d_{m} \exp \left(i 2 \pi m x^{\prime}\right),
$$

with 


$$
d_{m}=c_{k m},
$$

and $x^{\prime}=k_{1} g_{1}(x, y)+k_{2} g_{2}(x, y)$ is the coordinate transformation that brings $p_{k_{1}, k_{2}}\left(x^{\prime}\right)$ into the geometric layout of the $\left(k_{1}, k_{2}\right)$ moiré. In other words, we obtain the following:

The fundamental moiré theorem (for the superposition of two curvilinear gratings): The $\left(k_{1}, k_{2}\right)$ moiré $m_{k_{1}, k_{2}}(\mathbf{x})$ in the superposition of two gratings $r_{1}(\mathbf{x})$ $=p_{1}\left[g_{1}(\mathbf{x})\right]$ and $r_{2}(\mathbf{x})=p_{2}\left[g_{2}(\mathbf{x})\right]$ is given by $m_{k_{1}, k_{2}}(\mathbf{x})$ $=p_{k_{1}, k_{2}}\left[g_{k_{1}, k_{2}}(\mathbf{x})\right]$, where

1. $p_{k_{1}, k_{2}}\left(x^{\prime}\right)$, the normalized periodic profile of the $\left(k_{1}, k_{2}\right)$ moiré, is given by

$$
p_{k_{1}, k_{2}}\left(x^{\prime}\right)=\operatorname{sub}_{k_{1}}\left[p_{1}\left(x^{\prime}\right)\right] * \operatorname{sub}_{k_{2}}\left[p_{2}\left(x^{\prime}\right)\right] .
$$

2. $g_{k_{1}, k_{2}}(\mathbf{x})$, the coordinate transformation that brings $p_{k_{1}, k_{2}}\left(x^{\prime}\right)$ into the $\left(k_{1}, k_{2}\right)$ moiré $m_{k_{1}, k_{2}}(\mathbf{x})$, is given by

$$
g_{k_{1}, k_{2}}(\mathbf{x})=k_{1} g_{1}(\mathbf{x})+k_{2} g_{2}(\mathbf{x}) \text {. }
$$

In other words, the geometric transformation $g_{k_{1}, k_{2}}(\mathbf{x})$ of the $\left(k_{1}, k_{2}\right)$ moiré is a weighted sum of the geometric transformations of the individual gratings, where the weighting coefficients are the moiré indices $k_{i}$.

Using less formal language, we may formulate this theorem as follows:

Result 3: The $\left(k_{1}, k_{2}\right)$ moiré $m_{k_{1}, k_{2}}(x, y)$ generated in the superposition of the two curvilinear gratings $r_{1}(x, y)=p_{1}\left[g_{1}(x, y)\right]$ and $r_{2}(x, y)=p_{2}\left[g_{2}(x, y)\right]$ can be seen from the image-domain point of view as the result of a three-stage process:

1. Normalization of the original curvilinear gratings by, in each of them, replacing $g_{i}(x, y)$ with $x^{\prime}$ [i.e., by applying to each of them the inverse coordinate transformation $\left.\left.g_{i}(x, y) \rightarrow x^{\prime}\right]\right)$ in order to straighten them into uncurved, normalized periodic gratings having identical periods $\mathbf{T}=(1,0)$.

2. $T$ convolution of the $k_{1}$-sub-Fourier series of the first normalized grating with the $k_{2}$-sub-Fourier series of the second normalized grating. This gives the uncurved, normalized periodic profile of the $\left(k_{1}, k_{2}\right)$ moiré, with the same period $\mathbf{T}=(1,0)$.

3. Bending this normalized periodic profile of the moire into the actual curvilinear geometric layout of the moiré by replacing $x^{\prime}$ with $k_{1} g_{1}(x, y)+k_{2} g_{2}(x, y)$ [i.e., by applying the nonlinear coordinate transformation $x^{\prime}$ $\left.\rightarrow k_{1} g_{1}(x, y)+k_{2} g_{2}(x, y)\right]$.

An interesting consequence of the above theorem is that to synthesize a $\left(k_{1}, k_{2}\right)$ moiré whose geometric layout is given by a certain required function $g(x, y)$, all that we have to do is to choose two original layers whose bending functions $g_{1}(x, y)$ and $g_{2}(x, y)$ satisfy the condition

$$
k_{1} g_{1}(x, y)+k_{2} g_{2}(x, y)=g(x, y) .
$$

In the case of a $(1,-1)$-moiré this condition is simplified into $^{18}$

$$
g_{1}(x, y)-g_{2}(x, y)=g(x, y) .
$$

In particular, it may also happen that the superposition gives a periodic moiré even when the original layers are curved. Using Eq. (14), we can say now exactly when the $\left(k_{1}, k_{2}\right)$ moiré in the superposition of two curvilinear gratings is periodic: This occurs iff the coordinate transformation $k_{1} g_{1}(x, y)+k_{2} g_{2}(x, y)$ is affine, namely,

$$
k_{1} g_{1}(x, y)+k_{2} g_{2}(x, y)=a x+b y+c .
$$

In the case of a $(1,-1)$ moiré this condition becomes

$$
g_{1}(x, y)-g_{2}(x, y)=a x+b y+c .
$$

Example 1: A periodic moiré that is generated by the lateral shift of two identical layers on top of each other: This kind of situation occurs when the bending function $g(x, y)$ (which is common to both layers) happens to have the property

$$
\begin{aligned}
\forall x_{1}, x_{2}, \quad g\left[\left(x+x_{1}\right), y\right]-g\left[\left(x-x_{2}\right), y\right] & \\
& =a_{0} x+b_{0} y+c_{0} .
\end{aligned}
$$

For instance, in the superposition of two identical, laterally shifted zone gratings whose bending function is $g(x, y)=a x^{2}+b y^{2}$ (see Fig. 5), we have

$$
\begin{aligned}
g\left[\left(x+x_{1}\right), y\right] & -g\left[\left(x-x_{2}\right), y\right] \\
= & {\left[a\left(x+x_{1}\right)^{2}+b y^{2}\right]-\left[a\left(x-x_{2}\right)^{2}+b y^{2}\right] } \\
= & a\left(x^{2}+2 x x_{1}+x_{1}^{2}-x^{2}+2 x x_{2}-x_{2}^{2}\right) \\
= & 2 a\left(x_{1}+x_{2}\right) x+a\left(x_{1}^{2}-x_{2}^{2}\right) .
\end{aligned}
$$

Note that the $(1,-1)$ moiré obtained in such cases remains periodic for any lateral shifts $x_{1}, x_{2}$ in the original layers.

Example 2: When the two layers have an identical bending function $g(x, y)$ that does not have the above property, we can still force the superposed layers to give a periodic moiré by slightly modifying one or even both of them. For example, if we slightly modify the bending function of the first layer into $g(x, y)+x / 8$, the difference $g_{1}(x, y)-g_{2}(x, y)$ now becomes $x / 8$ and the moiré obtained in their superposition consists of periodic vertical bands [Fig. 6(a)]. However, unlike in the case of example 1 , the $(1,-1)$ moire in this case is periodic only when the two layers are superposed center on center without any shifts; the slightest shift (or rotation) between the two layers will destroy the periodicity of this moiré, as shown in Figs. 6(b) and 6(c).

Remark: The geometric layout of the curvilinear $\left(k_{1}, k_{2}\right)$ moiré in the $x, y$ plane, i.e., the locus of the centers of its curvilinear corrugations in the $x, y$ plane, is determined by the coordinate transformation $g_{k_{1}, k_{2}}(\mathbf{x})$. In other words, the reflectance (or transmittance) function of the isolated $\left(k_{1}, k_{2}\right)$ moire is constant along the curves $g_{k_{1}, k_{2}}(\mathbf{x})=$ constant. A general notion about the geometric layout of the $\left(k_{1}, k_{2}\right)$ moiré can already be obtained from the first harmonic term of the curvilinear Fourier-series development of $m_{k_{1}, k_{2}}(x, y)$, $\cos \left[2 \pi g_{k_{1}, k_{2}}(x, y)\right]$, by drawing the locus of its maxima, namely, the curves $g_{k_{1}, k_{2}}(x, y)=m, m \in \mathbb{Z}$, where the cosine $=1$.

The generalization of results 2 and 3 and of the fundamental moiré theorem to any $\left(k_{1}, \ldots, k_{m}\right)$ moiré between $m$ superposed curvilinear gratings are straightforward. 


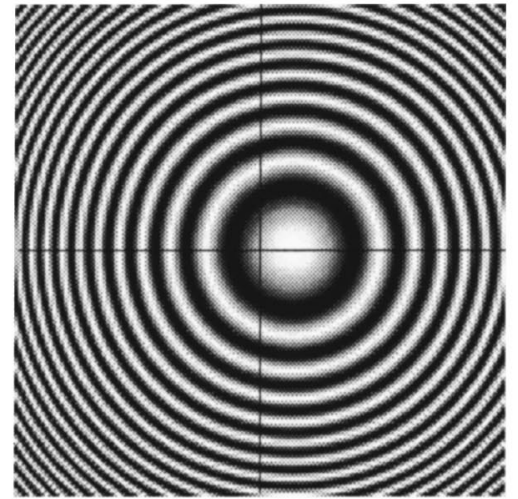

(a)

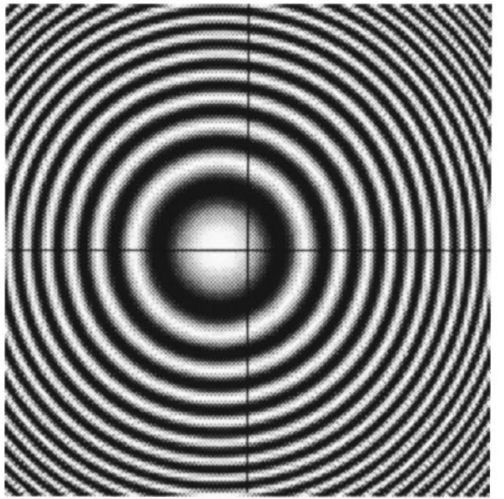

(b)

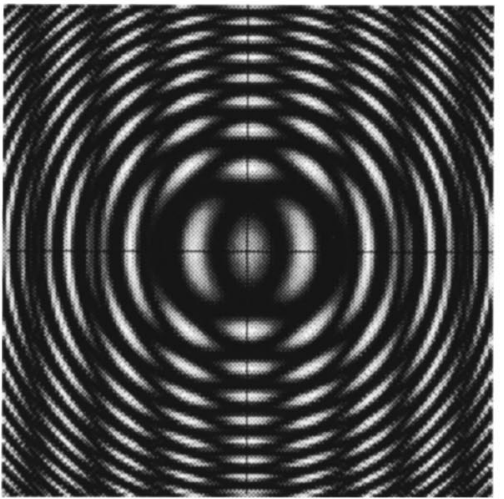

(c)

Fig. 5. Two circular zone gratings with raised cosinusoidal periodic profiles $(1 / 2) \cos (2 \pi f r)+1 / 2$, which have been horizontally shifted from the origin to the points $x=1(\mathrm{a}), x=-1(\mathrm{~b})$, and their superposition (c). The $(1,-1)$ moire is clearly seen in the superposition (c) in the form of periodic vertical bands.

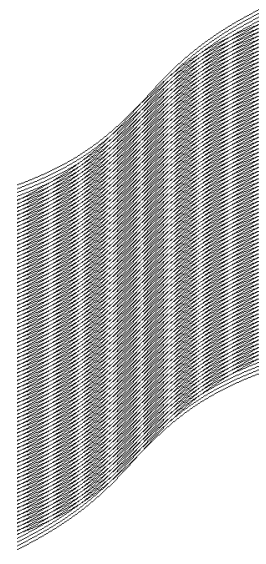

(a)

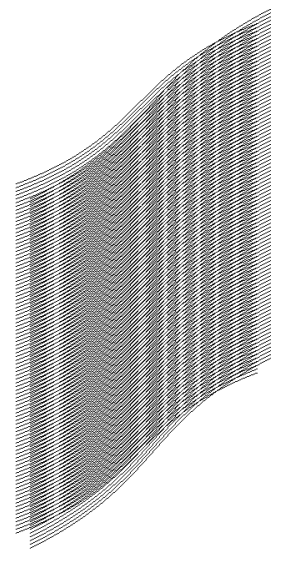

(b)

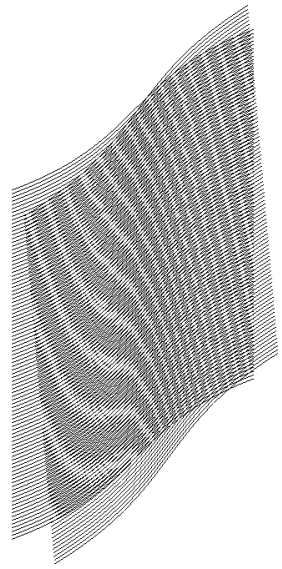

(c)

Fig. 6. Superposition of two curvilinear gratings whose bending functions are $g_{1}(x, y)=\arg \sinh (x)+x / 8$ and $g_{2}(x, y)=\arg \sinh (x) \operatorname{so}$ that $g_{1}(x, y)-g_{2}(x, y)=x / 8$. The $(1,-1)$ moiré obtained in their superposition consists of periodic vertical bands as shown in (a). However, this moiré is periodic only when the two layers are superposed center on center, and the slightest shift or rotation between the two layers destroys the periodicity of the moiré, as shown in (b) and (c). Note that the moiré bands seem to be darker in the center of each drawing; this happens because, for practical reasons, the curvilinear gratings were drawn here with a constant linewidth [compare with the correct, varying linewidths in Fig. 2(b)]. (Weak parasite moirés are a result of the production process.)

\section{B. Moiré Extraction in the Superposition of Curved Dot} Screens

The $\left(k_{1}, k_{2}, k_{3}, k_{4}\right)$ moiré generated between two superposed dot screens (see Section 4) is a case of particular interest. Owing to its special importance, this case will now be analyzed separately, although it is already covered by the above generalization taking $m=4$.

We start by presenting the 2D $T$-convolution theorem in terms of Fourier series:

$2 D$ T-convolution theorem: Let $p_{1}(x, y)$ and $p_{2}(x, y)$ be doubly periodic functions of the period $T_{x}, T_{y}$ integrable on a one-period interval $\left(0 \leqslant x \leqslant T_{x}, 0 \leqslant y\right.$ $\leqslant T_{y}$ ), and let their Fourier series representations be

$$
\begin{aligned}
p_{1}(x, y)= & \sum_{m=-\infty}^{\infty} \sum_{n=-\infty}^{\infty} c_{m, n}^{(1)} \\
& \times \exp \left[i 2 \pi\left(m x / T_{x}+n y / T_{y}\right)\right], \\
p_{2}(x, y)= & \sum_{m=-\infty}^{\infty} \sum_{n=-\infty}^{\infty} c_{m, n}^{(2)} \\
& \times \exp \left[i 2 \pi\left(m x / T_{x}+n y / T_{y}\right)\right] .
\end{aligned}
$$

Then their $T$ convolution

$$
\begin{aligned}
h(x, y)= & p_{1}(x, y) * * p_{2}(x, y) \\
= & \frac{1}{T_{x} T_{y}} \iint_{T_{x} T_{y}} p_{1}\left(x-x^{\prime}, y-y^{\prime}\right) \\
& \quad \times p_{2}\left(x^{\prime}, y^{\prime}\right) \mathrm{d} x^{\prime} \mathrm{d} y^{\prime}
\end{aligned}
$$

is also periodic with the same period $T_{x}, T_{y}$, and its Fourier series representation is

$$
\begin{aligned}
h(x, y)= & \sum_{m=-\infty}^{\infty} \sum_{n=-\infty}^{\infty} c_{m, n}^{(1)} c_{m, n}^{(2)} \\
& \times \exp \left[i 2 \pi\left(m x / T_{x}+n y / T_{y}\right)\right] .
\end{aligned}
$$

Using this Fourier series formalism, we now derive the counterpart of result 1 for the case of curved dot screens: Let $r_{1}(x, y)$ and $r_{2}(x, y)$ be curved dot screens. We have, therefore, 


$$
\begin{aligned}
r_{1}(x, y)= & p_{1}\left[g_{1}(x, y), g_{2}(x, y)\right] \\
= & \sum_{m=-\infty} \sum_{n=-\infty}^{\infty} c_{m, n}^{(1)} \\
& \quad \times \exp \left\{i 2 \pi\left[m g_{1}(x, y)+n g_{2}(x, y)\right]\right\}, \\
r_{2}(x, y)= & p_{2}\left[g_{3}(x, y), g_{4}(x, y)\right] \\
= & \sum_{m=-\infty}^{\infty} \sum_{n=-\infty}^{\infty} c_{m, n}^{(2)} \\
& \times \exp \left\{i 2 \pi\left[m g_{3}(x, y)+n g_{4}(x, y)\right]\right\},
\end{aligned}
$$

where $p_{1}\left(x^{\prime}, y^{\prime}\right)=\sum_{m=-\infty}^{\infty} \Sigma_{n=-\infty}^{\infty} c_{m, n}^{(1)} \exp \left[i 2 \pi\left(m x^{\prime}+n y^{\prime}\right)\right]$ and $\quad p_{2}\left(x^{\prime}, y^{\prime}\right)=\sum_{m=-\infty}^{\infty} \Sigma_{n=-\infty}^{\infty} c_{m, n}^{(2)} \exp \left[i 2 \pi\left(m x^{\prime}+n y^{\prime}\right)\right]$ are the periodic profiles of the dot screens $r_{1}(x, y)$ and $r_{2}(x, y)$, normalized to a $2 \mathrm{D}$ period of $\left(T_{x^{\prime}}, T_{y^{\prime}}\right)$ $=(1,1)$, and

$$
\left(\begin{array}{l}
x^{\prime} \\
y^{\prime}
\end{array}\right)=\left(\begin{array}{l}
g_{1}(x, y) \\
g_{2}(x, y)
\end{array}\right), \quad\left(\begin{array}{l}
x^{\prime} \\
y^{\prime}
\end{array}\right)=\left(\begin{array}{l}
g_{3}(x, y) \\
g_{4}(x, y)
\end{array}\right)
$$

[or in vector notation $\mathbf{x}^{\prime}=\mathbf{g}_{1}(\mathbf{x})$ and $\mathbf{x}^{\prime}=\mathbf{g}_{2}(\mathbf{x})$ ] are the nonlinear coordinate transformations that transform $p_{1}\left(x^{\prime}, y^{\prime}\right)$ and $p_{2}\left(x^{\prime}, y^{\prime}\right)$ into the curved dot screens $r_{1}(x, y)$ and $r_{2}(x, y)$, respectively.

As we have seen in Section 4 [see Eq. (8)], the superposition of the two dot screens is expressed by the product

$r_{1}(x, y) r_{2}(x, y)$

$$
\begin{aligned}
& =\sum_{n_{1}=-\infty}^{\infty} \sum_{n_{2}=-\infty}^{\infty} \sum_{n_{3}=-\infty}^{\infty} \sum_{n_{4}=-\infty}^{\infty} c_{n_{1}, n_{2}}^{(1)} c_{n_{3}, n_{4}}^{(2)} \\
& \quad \times \exp \left\{i 2 \pi \left[n_{1} g_{1}(x, y)+n_{2} g_{2}(x, y)\right.\right. \\
& \left.\left.\quad+n_{3} g_{3}(x, y)+n_{4} g_{4}(x, y)\right]\right\},
\end{aligned}
$$

and the partial sum that corresponds to the $(1,0,-1,0)$ moire consists of all the terms of this double sum whose indices are $m, n,-m$, and $-n$, namely [see Eq. (10],

$$
\begin{aligned}
& m_{1,0,-1,0}(x, y) \\
& =\sum_{m=-\infty}^{\infty} \sum_{n=-\infty}^{\infty} c_{m, n}^{(1)} c_{-m,-n}^{(2)} \exp \left(i 2 \pi \left\{m \left[g_{1}(x, y)\right.\right.\right. \\
& \left.\left.\left.\quad-g_{3}(x, y)\right]+n\left[g_{2}(x, y)-g_{4}(x, y)\right]\right\}\right) .
\end{aligned}
$$

Let us denote by $p_{1,0,-1,0}\left(x^{\prime}, y^{\prime}\right)$ the periodic profile [normalized to the $2 \mathrm{D}$ period $\left(T_{x^{\prime}}, T_{y^{\prime}}\right)=(1,1)$ ] of $m_{1,0,-1,0}(x, y)$ :

$$
\begin{aligned}
p_{1,0,-1,0}\left(x^{\prime}, y^{\prime}\right)= & \sum_{m=-\infty}^{\infty} \sum_{n=-\infty}^{\infty} c_{m, n}^{(1)} c_{-m,-n}^{(2)} \\
& \times \exp \left[i 2 \pi\left(m x^{\prime}+n y^{\prime}\right)\right] .
\end{aligned}
$$

We see, therefore, by the $2 \mathrm{D} T$-convolution theorem that $p_{1,0,-1,0}\left(x^{\prime}, y^{\prime}\right)$ is simply the $2 \mathrm{D} T$ convolution

$$
p_{1,0,-1,0}\left(x^{\prime}, y^{\prime}\right)=p_{1}\left(x^{\prime}, y^{\prime}\right) * * p_{2}\left(-x^{\prime},-y^{\prime}\right),
$$

where

$$
\left(\begin{array}{l}
x^{\prime} \\
y^{\prime}
\end{array}\right)=\left(\begin{array}{l}
g_{1}(x, y)-g_{3}(x, y) \\
g_{2}(x, y)-g_{4}(x, y)
\end{array}\right),
$$

or, in vector notation, $\mathbf{x}^{\prime}=\mathbf{g}_{1}(\mathbf{x})-\mathbf{g}_{2}(\mathbf{x})$, is the $2 \mathrm{D}$ coordinate transformation that brings $p_{1,0,-1,0}\left(x^{\prime}, y^{\prime}\right)$ into the geometric layout of the $(1,0,-1,0)$ moiré. In other words, we obtained the following result:

Result 4: The $(1,0,-1,0)$ moiré $m_{1,0,-1,0}(\mathbf{x})$ in the superposition of two curved dot screens $r_{1}(\mathbf{x})=p_{1}\left[\mathbf{g}_{1}(\mathbf{x})\right]$ and $r_{2}(\mathbf{x})=p_{2}\left[\mathbf{g}_{2}(\mathbf{x})\right]$ is given by $m_{1,0,-1,0}(\mathbf{x})$ $=p_{1,0,-1,0}\left[\mathbf{g}_{1,0,-1,0}(\mathbf{x})\right]$, where

1. $p_{1,0,-1,0}(\mathbf{x})$, the normalized periodic profile of the ( $1,0,-1,0)$ moiré, is the $T$ convolution of the normalized periodic profiles of the original dot-screens:

$$
p_{1,0,-1,0}\left(\mathbf{x}^{\prime}\right)=p_{1}\left(\mathbf{x}^{\prime}\right) * * p_{2}\left(-\mathbf{x}^{\prime}\right)
$$

2. $\mathbf{g}_{1,0,-1,0}(\mathbf{x})$, the bending transformation of the $(1,0,-1,0)$ moiré, is given by

$$
\mathbf{g}_{1,0,-1,0}(\mathbf{x})=\mathbf{g}_{1}(\mathbf{x})-\mathbf{g}_{2}(\mathbf{x}) .
$$

More generally, we have seen in Section 4 that the partial sum of (15) that corresponds to the $\left(k_{1}, k_{2}, k_{3}, k_{4}\right)$ moiré consists of all the terms of the double sum whose indices are $m k_{1}-n k_{2}, m k_{2}+n k_{1}, m k_{3}-n k_{4}$, and $m k_{4}+n k_{3}$, namely [see Eq. (9)],

$$
\begin{aligned}
& m_{k_{1}, k_{2}, k_{3}, k_{4}}(x, y) \\
& =\sum_{m=-\infty}^{\infty} \sum_{n=-\infty}^{\infty} c_{m k_{1}-n k_{2}, m k_{2}+n k_{1}}^{(1)} c_{m k_{3}-n k_{4}, m k_{4}+n k_{3}}^{(2)} \\
& \quad \times \exp \left(i 2 \pi \left\{m \left[k_{1} g_{1}(x, y)+k_{2} g_{2}(x, y)\right.\right.\right. \\
& \left.\quad+k_{3} g_{3}(x, y)+k_{4} g_{4}(x, y)\right]+n\left[-k_{2} g_{1}(x, y)\right. \\
& \left.\left.\left.\quad+k_{1} g_{2}(x, y)-k_{4} g_{3}(x, y)+k_{3} g_{4}(x, y)\right]\right\}\right) .
\end{aligned}
$$

If we denote by $p_{k_{1}, k_{2}, k_{3}, k_{4}}\left(x^{\prime}, y^{\prime}\right)$ the periodic profile of $m_{k_{1}, k_{2}, k_{3}, k_{4}}(x, y)$ [normalized to the 2D period $\left.\left(T_{x^{\prime}}, T_{y^{\prime}}\right)=(1,1)\right]$,

$$
\begin{aligned}
p_{k_{1}, k_{2}, k_{3}, k_{4}}\left(x^{\prime}, y^{\prime}\right) & \\
= & \sum_{m=-\infty}^{\infty} \sum_{n=-\infty}^{\infty} c_{m k_{1}-n k_{2}, m k_{2}+n k_{1}}^{(1)} c_{m k_{3}-n k_{4}, m k_{4}+n k_{3}}^{(2)} \\
& \times \exp \left[i 2 \pi\left(m x^{\prime}+n y^{\prime}\right)\right]
\end{aligned}
$$

then we see by the $2 \mathrm{D} T$-convolution theorem that $p_{k_{1}, k_{2}, k_{3}, k_{4}}\left(x^{\prime}, y^{\prime}\right)$ is simply the 2D $T$ convolution

$$
\begin{aligned}
& p_{k_{1}, k_{2}, k_{3}, k_{4}}\left(x^{\prime}, y^{\prime}\right) \\
& \quad=\operatorname{sub}_{k_{1}, k_{2}}\left[p_{1}\left(x^{\prime}, y^{\prime}\right)\right] * * \operatorname{sub}_{k_{3}, k_{4}}\left[p_{2}\left(x^{\prime}, y^{\prime}\right)\right],
\end{aligned}
$$

where $\operatorname{sub}_{r, s}\left[p\left(x^{\prime}, y^{\prime}\right)\right]$ is the $(r, s)$-sub-Fourier series of $p\left(x^{\prime}, y^{\prime}\right)$, i.e., the periodic function [with 2D period $\left(T_{x^{\prime}}, T_{y^{\prime}}\right)=(1,1)$ ] whose Fourier series contains only every $(r, s)$ th coefficient from the 2D Fourier series $p\left(x^{\prime}, y^{\prime}\right)=\sum_{m=-\infty}^{\infty} \sum_{n=-\infty}^{\infty} c_{m, n} \exp \left[i 2 \pi\left(m x^{\prime}+n y^{\prime}\right)\right]$ :

$$
\begin{aligned}
\operatorname{sub}_{r, s}\left[p\left(x^{\prime}, y^{\prime}\right)\right]= & \sum_{m=-\infty}^{\infty} \sum_{n=-\infty}^{\infty} d_{m, n} \\
& \times \exp \left[i 2 \pi\left(m x^{\prime}+n y^{\prime}\right)\right],
\end{aligned}
$$

with

and where 


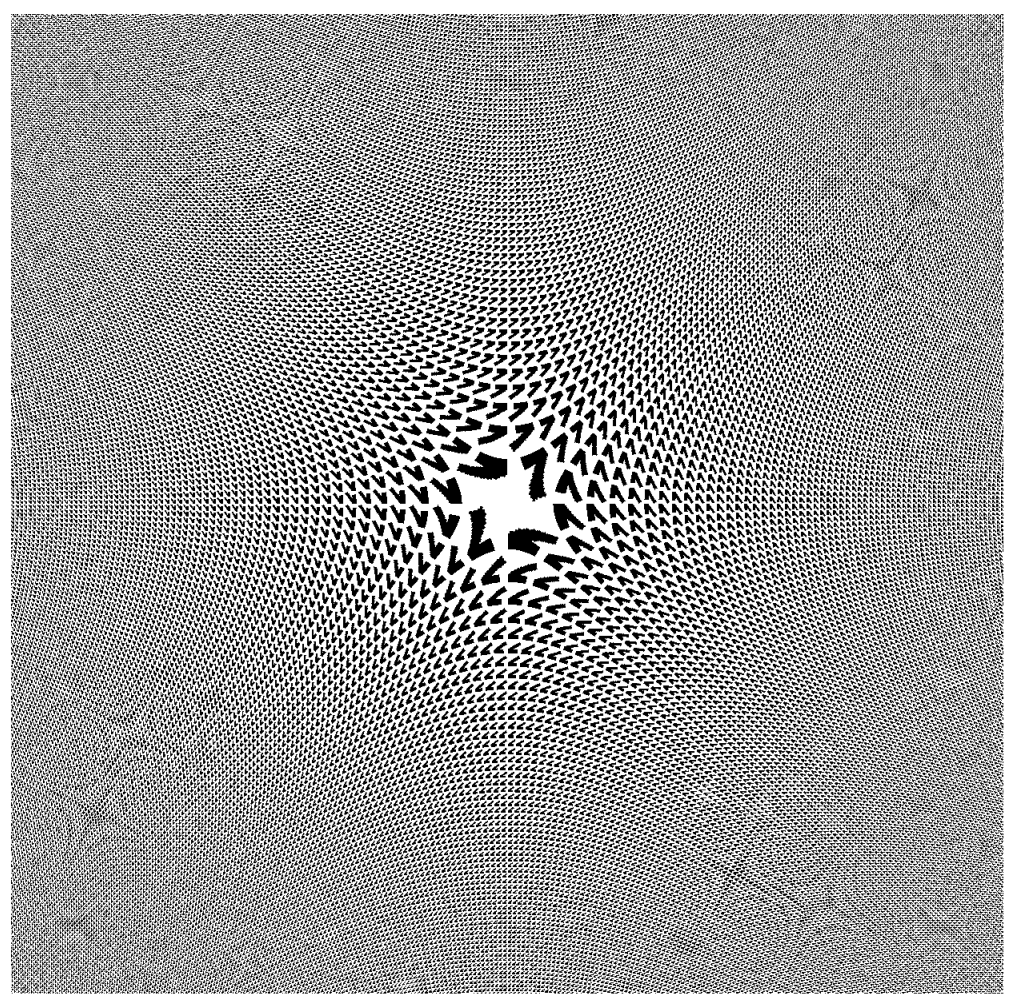

(a)

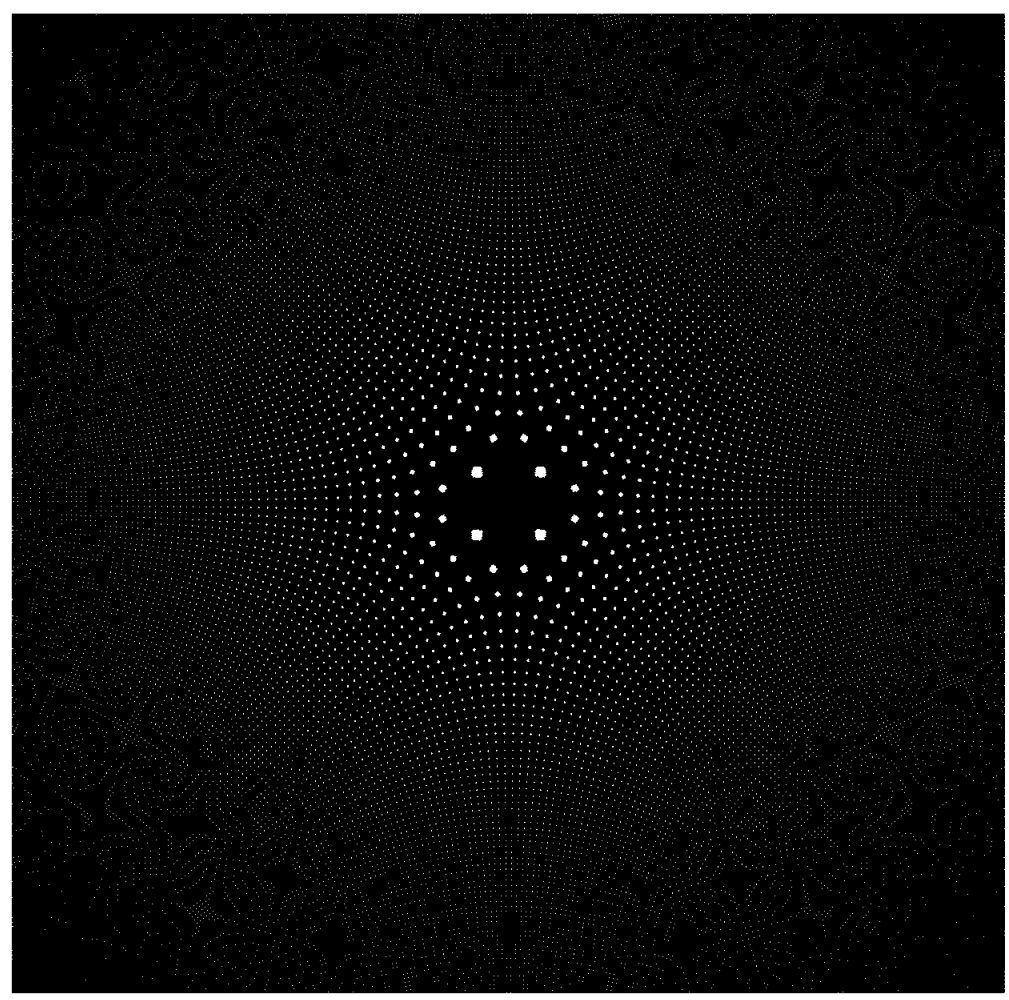

(b)

Fig. 7. Continued on facing page.

$\left(\begin{array}{l}x^{\prime} \\ y^{\prime}\end{array}\right)=\left(\begin{array}{c}k_{1} g_{1}(x, y)+k_{2} g_{2}(x, y)+k_{3} g_{3}(x, y)+k_{4} g_{4}(x, y) \\ -k_{2} g_{1}(x, y)+k_{1} g_{2}(x, y)-k_{4} g_{3}(x, y)+k_{3} g_{4}(x, y)\end{array}\right)$

is the $2 \mathrm{D}$ coordinate transformation that brings $p_{k_{1}, k_{2}, k_{3}, k_{4}}\left(x^{\prime}, y^{\prime}\right)$ into the geometric layout of the
( $\left.k_{1}, k_{2}, k_{3}, k_{4}\right)$ moiré. Note that this bending transformation can be rearranged in the form

$$
\left(\begin{array}{l}
x^{\prime} \\
y^{\prime}
\end{array}\right)=\left[\begin{array}{cc}
k_{1} & k_{2} \\
-k_{2} & k_{1}
\end{array}\right]\left(\begin{array}{l}
g_{1}(x, y) \\
g_{2}(x, y)
\end{array}\right)+\left[\begin{array}{cc}
k_{3} & k_{4} \\
-k_{4} & k_{3}
\end{array}\right]\left(\begin{array}{l}
g_{3}(x, y) \\
g_{4}(x, y)
\end{array}\right),
$$




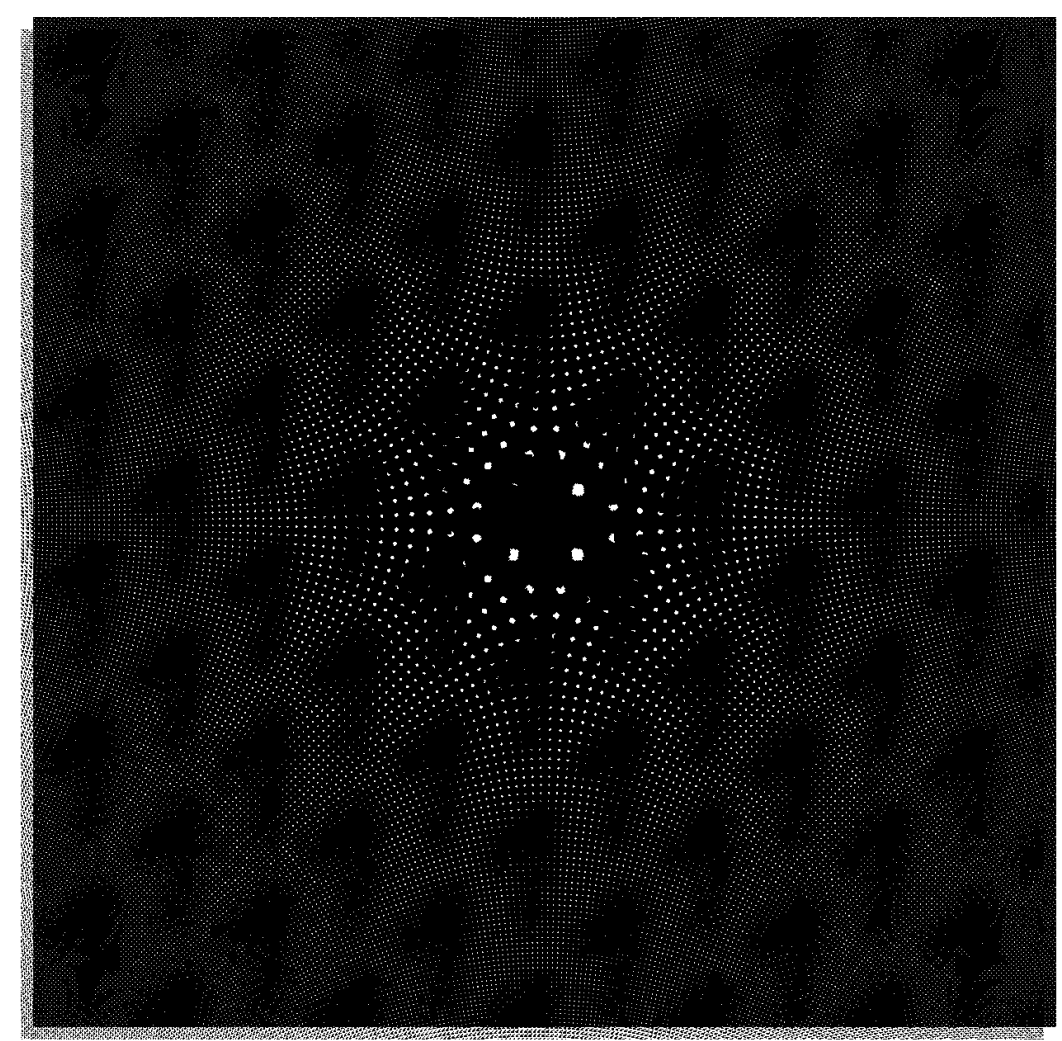

(c)

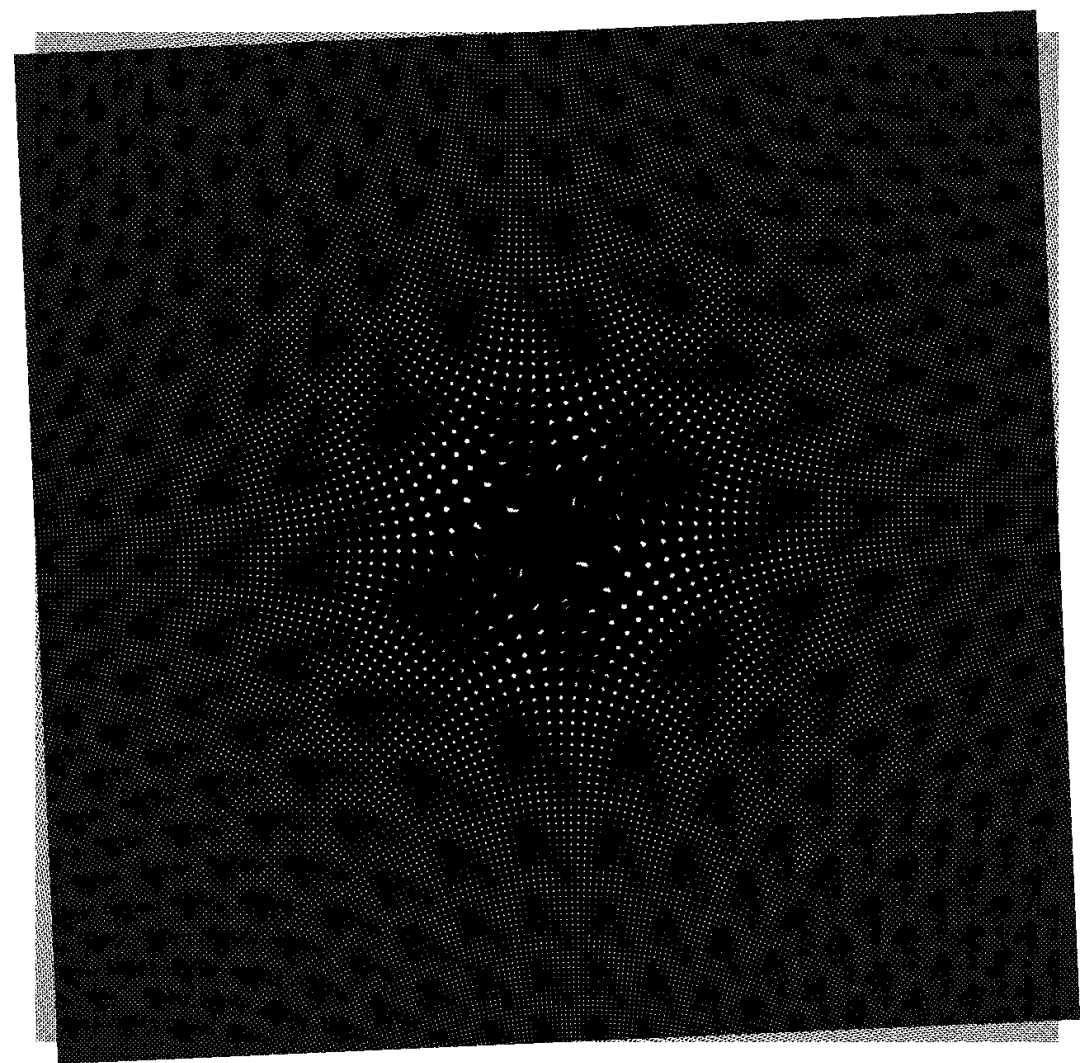

(d)

Fig. 7. Dot-screen superposition illustrating example 1 of Section 5.B. (a) Curved dot screen $r_{1}(x, y)$ consisting of distorted 1's, (b) curved dot screen $r_{2}(x, y)$ consisting of small pinholes. The two layers have been distorted by the same nonlinear coordinate transformation $\mathbf{g}(x, y)=\left(2 x y, x^{2}-y^{2}\right)$. As shown in (c), the $(1,0,-1,0)$ moiré generated when $r_{2}(x, y)$ is laterally shifted on top of $r_{1}(x, y)$ is purely periodic, although both $r_{1}(x, y)$ and $r_{2}(x, y)$ are not periodic; this periodic moiré consists of a screen of magnified 1's, whose period and orientation depend on the shift. Note that rotations destroy the periodicity of the moiré, as illustrated in (d). 
from which we obtain its vector form

$$
\mathbf{x}^{\prime}=\mathrm{K}_{1} \mathbf{g}_{1}(\mathbf{x})+\mathrm{K}_{2} \mathbf{g}_{2}(\mathbf{x}),
$$

where $\mathrm{K}_{1}$ and $\mathrm{K}_{2}$ denote the matrices $\left[\begin{array}{cc}k_{1} & k_{2} \\ -k_{2} & k_{1}\end{array}\right]$ and $\left[\begin{array}{cc}k_{3} & k_{4} \\ -k_{4} & k_{3}\end{array}\right]$, respectively. We therefore obtain the following:

The fundamental moire theorem (for the superposition of two curved dot screens): The $\left(k_{1}, k_{2}, k_{3}, k_{4}\right)$ moiré $m_{k_{1}, k_{2}, k_{3}, k_{4}}(\mathbf{x})$ in the superposition of two curved dot screens $r_{1}(\mathbf{x})=p_{1}\left[\mathbf{g}_{1}(\mathbf{x})\right]$ and $r_{2}(\mathbf{x})=p_{2}\left[\mathbf{g}_{2}(\mathbf{x})\right]$ is given by $m_{k_{1}, k_{2}, k_{3}, k_{4}}(\mathbf{x})=p_{k_{1}, k_{2}, k_{3}, k_{4}}\left[\mathbf{g}_{k_{1}, k_{2}, k_{3}, k_{4}}(\mathbf{x})\right]$, where

1. $p_{k_{1}, k_{2}, k_{3}, k_{4}}(\mathbf{x})$, the normalized periodic profile of the $\left(k_{1}, k_{2}, k_{3}, k_{4}\right)$ moiré, is given by

$$
p_{k_{1}, k_{2}, k_{3}, k_{4}}\left(\mathbf{x}^{\prime}\right)=\operatorname{sub}_{k_{1}, k_{2}}\left[p_{1}\left(\mathbf{x}^{\prime}\right)\right] * * \operatorname{sub}_{k_{3}, k_{4}}\left[p_{2}\left(\mathbf{x}^{\prime}\right)\right] \text {; }
$$

2. $\mathbf{g}_{k_{1}, k_{2}, k_{3}, k_{4}}(\mathbf{x})$, the bending transformation of the $\left(k_{1}, k_{2}, k_{3}, k_{4}\right)$ moiré, is given by

$$
\mathbf{g}_{k_{1}, k_{2}, k_{3}, k_{4}}(\mathbf{x})=\mathrm{K}_{1} \mathbf{g}_{1}(\mathbf{x})+\mathrm{K}_{2} \mathbf{g}_{2}(\mathbf{x}) \text {. }
$$

The generalization of this theorem to any $\left(k_{1}, \ldots, k_{2 m}\right)$ moire in the superposition of $m$ curved dot screens is straightforward.

We obtain, therefore, as an immediate consequence of this theorem,

Result 5: The geometric layout of the moire (which is determined by its bending transformation) and the periodic profile of the moiré are completely independent of each other: The geometric layout of the moiré is influenced only by the geometric layout of the superposed layers, and the periodic profile of the moire depends only on the periodic profiles of the superposed layers.

Using less formal language than in the theorem, we can now state the counterpart of result 3 for the superposition of two curved dot screens as follows:

Result 6: Let $r_{1}(x, y)$ and $r_{2}(x, y)$ be two curved dot screens, which are obtained from two twofold periodic dot screens by the nonlinear coordinate transformations

$$
\mathbf{g}_{1}:\left(\begin{array}{l}
x \\
y
\end{array}\right) \rightarrow\left(\begin{array}{l}
g_{1}(x, y) \\
g_{2}(x, y)
\end{array}\right), \quad \mathbf{g}_{2}:\left(\begin{array}{l}
x \\
y
\end{array}\right) \rightarrow\left(\begin{array}{l}
g_{3}(x, y) \\
g_{4}(x, y)
\end{array}\right),
$$

respectively. The $\left(k_{1}, k_{2}, k_{3}, k_{4}\right)$ moiré $m_{k_{1}, k_{2}, k_{3}, k_{4}}(x, y)$ generated in the superposition of these curved dot screens can be seen from the image-domain point of view as the result of a three-stage process:

1. Normalization of the original curved dot screens by, in each of them, replacing $\left[g_{i}(x, y), g_{i+1}(x, y)\right]$ with $\left(x^{\prime}, y^{\prime}\right)$ \{i.e., by applying to each of them the inverse coordinate transformation $\quad\left[g_{i}(x, y), g_{i+1}(x, y)\right]$ $\left.\rightarrow\left(x^{\prime}, y^{\prime}\right)\right\}$ in order to straighten them into uncurved, normalized $2 \mathrm{D}$ periodic dot screens that have identical pe$\operatorname{riods}\left(T_{x^{\prime}}, T_{y^{\prime}}\right)=(1,1)$.

2. T-convolution of the $2 \mathrm{D}\left(k_{1}, k_{2}\right)$-sub-Fourier series of the first normalized dot screen with the $2 \mathrm{D}$ $\left(k_{3}, k_{4}\right)$-sub-Fourier series of the second normalized dot screen. This gives the uncurved, normalized periodic profile of the $\left(k_{1}, k_{2}, k_{3}, k_{4}\right)$ moiré, with the same pe$\operatorname{riod}\left(T_{x^{\prime}}, T_{y^{\prime}}\right)=(1,1)$.

3. Bending this normalized periodic profile of the moire into the actual curved geometric layout of the moiré, by replacing $\left(x^{\prime}, y^{\prime}\right)$ with $\left[k_{1} g_{1}(x, y)\right.$ $+k_{2} g_{2}(x, y)+k_{3} g_{3}(x, y)+k_{4} g_{4}(x, y), \quad-k_{2} g_{1}(x, y)$ $\left.+k_{1} g_{2}(x, y)-k_{4} g_{3}(x, y)+k_{3} g_{4}(x, y)\right]$, i.e., by applying the nonlinear coordinate transformation

$$
\left(\begin{array}{l}
x^{\prime} \\
y^{\prime}
\end{array}\right) \rightarrow\left(\begin{array}{c}
k_{1} g_{1}(x, y)+k_{2} g_{2}(x, y)+k_{3} g_{3}(x, y)+k_{4} g_{4}(x, y) \\
-k_{2} g_{1}(x, y)+k_{1} g_{2}(x, y)-k_{4} g_{3}(x, y)+k_{3} g_{4}(x, y)
\end{array}\right) .
$$

It is interesting to note, as we have already seen for curvilinear gratings, that in certain cases the coordinate transformation in step (3) may give a $2 \mathrm{D}$ periodic moiré even when the original layers are curved, i.e., when the coordinate transformations $g_{i}(x, y)$ of the individual layers are not linear. In the particular case of the $(1,0,-1,0)$ moiré (see result 4$)$, where the coordinate transformation of step (3) is simplified into

$$
\left(\begin{array}{l}
x^{\prime} \\
y^{\prime}
\end{array}\right) \rightarrow\left(\begin{array}{l}
g_{1}(x, y)-g_{3}(x, y) \\
g_{2}(x, y)-g_{4}(x, y)
\end{array}\right)
$$

this happens iff the coordinate transformation (18) is an affine transformation, namely,

$$
\begin{aligned}
& g_{1}(x, y)-g_{3}(x, y)=a_{1} x+b_{1} y+c_{1}, \\
& g_{2}(x, y)-g_{4}(x, y)=a_{2} x+b_{2} y+c_{2} .
\end{aligned}
$$

Example 1: Let $p_{1}\left(x^{\prime}, y^{\prime}\right)$ be a periodic dot screen whose period consists of the digit 1 , and let $r_{1}(x, y)$ be the curved dot screen obtained by applying to $p_{1}\left(x^{\prime}, y^{\prime}\right)$ the coordinate transformation

$$
\left(\begin{array}{l}
x^{\prime} \\
y^{\prime}
\end{array}\right)=\left(\begin{array}{c}
2 x y \\
x^{2}-y^{2}
\end{array}\right) .
$$

If we laterally shift on top of this curved dot screen a second dot screen that was subject to the same coordinate transformation, we obtain a twofold periodic moiré since

$$
\begin{aligned}
{\left[2\left(x+x_{0}\right) y\right]-\left[2\left(x-x_{0}\right) y\right] } & =4 x_{0} y, \\
{\left[\left(x+x_{0}\right)^{2}-y^{2}\right]-\left[\left(x-x_{0}\right)^{2}-y^{2}\right] } & =4 x_{0} x .
\end{aligned}
$$

Now, if the second layer consists of small pinholes, we obtain in the superposition a periodic $(1,0,-1,0)$ moiré whose normalized periodic profile is, according to result 4 , a $T$ convolution of the shape of 1 with the pinhole, which gives again a 1-shaped periodic profile. We obtain therefore a periodic $(1,0,-1,0)$ moiré whose period consists of a magnified digit 1 , even though the two superposed screens are not periodic. This is illustrated in Fig. 7.

More generally, to synthesize a $\left(k_{1}, k_{2}, k_{3}, k_{4}\right)$ moiré whose geometric layout is given by the two independent functions $g^{(1)}(x, y)$ and $g^{(2)}(x, y)$, all that we have to do is to choose two original layers whose bending functions $g_{1}(x, y), g_{2}(x, y), g_{3}(x, y)$, and $g_{4}(x, y)$ satisfy the condition

$$
\begin{array}{r}
k_{1} g_{1}(x, y)+k_{2} g_{2}(x, y)+k_{3} g_{3}(x, y)+k_{4} g_{4}(x, y) \\
=g^{(1)}(x, y), \\
-k_{2} g_{1}(x, y)+k_{1} g_{2}(x, y)-k_{4} g_{3}(x, y)+k_{3} g_{4}(x, y) \\
=g^{(2)}(x, y) .
\end{array}
$$

In the case of a $(1,0,-1,0)$ moiré, this condition is simplified into 


$$
\begin{aligned}
& g_{1}(x, y)-g_{3}(x, y)=g^{(1)}(x, y), \\
& g_{2}(x, y)-g_{4}(x, y)=g^{(2)}(x, y) .
\end{aligned}
$$

The periodic profile of the synthesized moiré will depend only on the periodic profiles of the superposed layers.

Finally, note that the second part of the fundamental moiré theorem remains true even when the original periodic layers $p_{1}\left(x^{\prime}, y^{\prime}\right)$ and $p_{2}\left(x^{\prime}, y^{\prime}\right)$ are not normalized and have different periods and orientations. This allows us to interpret the second part of the theorem as follows:

Result 7: When the two original, uncurved periodic layers $p_{1}\left(\mathbf{x}^{\prime}\right)$ and $p_{2}\left(\mathbf{x}^{\prime}\right)$ are transformed into curved layers $r_{1}(\mathbf{x})=p_{1}\left[\mathbf{g}_{1}(\mathbf{x})\right]$ and $r_{2}(\mathbf{x})=p_{2}\left[\mathbf{g}_{2}(\mathbf{x})\right]$ by transformations $\mathbf{g}_{1}(\mathbf{x})$ and $\mathbf{g}_{2}(\mathbf{x})$, respectively, the periodic $\left(k_{1}, k_{2}, k_{3}, k_{4}\right)$ moiré between the original noncurved layers is transformed into a curved moiré by the geometric transformation $\mathbf{g}_{k_{1}, k_{2}, k_{3}, k_{4}}(\mathbf{x})=\mathbf{K}_{1} \mathbf{g}_{1}(\mathbf{x})$ $+\mathrm{K}_{2} \mathbf{g}_{2}(\mathbf{x})$.

\section{CONCLUSIONS}

In this paper we explore the moiré effects generated in the superposition of geometrically transformed periodic structures (repetitive structures). We show that the geometric transformation undergone by any $\left(k_{1}, \ldots, k_{n}\right)$ moiré between repetitive structures is a weighted sum of the geometric transformations of the individual layers, where the weighting coefficients are given by the moire indices $k_{i}$. The intensity profile of the moiré, for its part, can be interpreted by virtue of the $T$-convolution theorem as a normalized $T$ convolution of the original superposed structures (or of the sub-Fourier series thereof). These results are first demonstrated for moirés in the superposition of curvilinear gratings and then for the more general 2D case of moirés between curved dot screens. These fundamental results offer a full quantitative and qualitative analysis of any moiré in the superposition of repetitive structures. In particular, we obtain the interesting result that the geometric layout and the periodic profile of the moiré are completely independent of each other; the geometric layout of the moiré is influenced only by the geometric layout of the superposed layers, and the periodic profile of the moire depends only on the periodic profiles of the superposed layers.

Moreover, in addition to the full analysis of the geometric layout and the intensity profile of any moiré in the superposition of repetitive layers, our results also enable us to synthesize moirés with any given geometric layout and with any required periodic profile. We show how one should design the original layers in order to obtain the desired moiré effect, and in particular we show how one may obtain a fully periodic moiré even if the original layers are repetitive but not necessarily periodic.

Finally, it should be noted that although for the sake of simplicity our results have been presented throughout this paper for the case of two superposed layers, our re- sults are general, and their extension to moiré effects between any $m$ superposed repetitive structures is straightforward.

\section{REFERENCES AND NOTES}

1. K. Patorski, Handbook of the Moiré Fringe Technique (Elsevier, Amsterdam, 1993).

2. O. Kafri and I. Glatt, The Physics of Moiré Metrology (Wiley, New York, 1989).

3. A. T. Shepherd, " 25 years of moiré fringe measurement," Precis. Eng. 1, 61-69 (1979).

4. H. Takasaki, "Moiré topography," Appl. Opt. 9, 1467-1472 (1970).

5. P. S. Theocaris, Moiré Fringes in Strain Analysis (Pergamon, Oxford, UK, 1969).

6. Y. Nishijima and G. Oster, "Moiré patterns: their application to refractive index and refractive index gradient measurements," J. Opt. Soc. Am. 54, 1-5 (1964).

7. G. Oster, M. Wasserman, and C. Zwerling, "Theoretical interpretation of moiré patterns," J. Opt. Soc. Am. 54, 169175 (1964).

8. O. Bryngdahl, "Moiré: formation and interpretation," J. Opt. Soc. Am. 64, 1287-1294 (1974).

9. O. Bryngdahl, "Moiré and higher grating harmonics," J. Opt. Soc. Am. 65, 685-694 (1975).

10. I. Amidror and R. D. Hersch, "Fourier-based analysis of phase shifts in the superposition of periodic layers and their moiré effects," J. Opt. Soc. Am. A 13, 974-987 (1996).

11. See, for example, K. Patorski, Handbook of the Moiré Fringe Technique (Elsevier, Amsterdam, 1993), pp. 16-21.

12. Note that the term curvature is defined in mathematics in a different way; see, for example, R. Courant, Differential and Integral Calculus (Wiley-Interscience, New York, 1988), Vol. II, p. 86.

13. The equivalence between a $2 \mathrm{D}$ mapping from $\mathrm{R}^{2}$ onto itself and a coordinate change in $R^{2}$ is discussed and illustrated in R. Courant, Differential and Integral Calculus (WileyInterscience, New York, 1988), Vol. II, pp. 133-140.

14. If $g_{1}(x, y)$ and $g_{2}(x, y)$ are dependent, for instance, $g_{2}(x, y)=g_{1}(x, y)^{2}$, then the 2D transformation $\mathbf{g}(x, y)$ is degenerate, and it maps $R^{2}$ into a $1 D$ curve in $\mathbb{R}^{2}$; see $R$. Courant, Differential and Integral Calculus (WileyInterscience, New York, 1988), Vol. II, p. 155.

15. See, for example, R. Courant, Differential and Integral Calculus (Wiley-Interscience, New York, 1988), Vol. II, pp. 154-155. If $g_{1}(x, y)$ and $g_{2}(x, y)$ satisfy also the Cauchy-Riemann conditions (a) $\partial g_{1} / \partial x=\partial g_{2} / \partial y, \partial g_{1} / \partial y$ $=-\partial g_{2} / \partial x \quad$ or $\quad$ (b) $\quad \partial g_{1} / \partial x=-\partial g_{2} / \partial y, \quad \partial g_{1} / \partial y$ $=-\partial g_{2} / \partial x$, then the transformation $\mathbf{g}(\mathbf{x})$ is conformal [see R. Courant, Differential and Integral Calculus (WileyInterscience, New York, 1988), Vol. II, pp. 166-167], and it maps the straight lines $x=$ constant, $y=$ constant into curve families $x^{\prime}=$ constant and $y^{\prime}=$ constant, which intersect at right angles. This orthogonality is clearly stronger than the mere independence of $g_{1}(x, y)$ and $g_{2}(x, y)$; and indeed, condition (a) implies $J(x, y)>0$, and condition (b) implies $J(x, y)<0$. Such an orthogonality is not required for our needs [see for instance Fig. 2(b)], but it is advantageous; for example, it guarantees that the two curvilinear gratings that together form our curved grid $r(x, y)$ do not generate moirés between themselves within the curved grid itself.

16. A. W. Lohmann and D. P. Paris, "Variable Fresnel zone pattern," Appl. Opt. 6, 1567-1570 (1967).

17. A. Zygmund, Trigonometric Series (Cambridge U. Press, Cambridge, UK, 1968), Vol. 1, p. 36.

18. Note that this particular case has already been proposed in Ref. 16. 